University of Nebraska - Lincoln DigitalCommons@University of Nebraska - Lincoln

U.S. Air Force Research

U.S. Department of Defense

2013

\title{
Challenges in the aerodynamics modeling of an oscillating and translating airfoil at large incidence angles
}

Mehdi Ghoreyshi

U.S. Air Force Academy, Mehdi.Ghoreyshi@usafa.edu

Russell M. Cummings

USAF Academy, russ.cummings@usafa.edu

Follow this and additional works at: http:// digitalcommons.unl.edu/usafresearch

Mehdi Ghoreyshi and Cummings, Russell M., "Challenges in the aerodynamics modeling of an oscillating and translating airfoil at large incidence angles" (2013). U.S. Air Force Research. 54.

http://digitalcommons.unl.edu/usafresearch/54

This Article is brought to you for free and open access by the U.S. Department of Defense at DigitalCommons@University of Nebraska - Lincoln. It has been accepted for inclusion in U.S. Air Force Research by an authorized administrator of DigitalCommons@University of Nebraska - Lincoln. 


\title{
Challenges in the aerodynamics modeling of an oscillating and translating airfoil at large incidence angles
}

\author{
Mehdi Ghoreyshi ${ }^{*, 1}$, Russell M. Cummings ${ }^{2}$ \\ Modeling and Simulation Research Center, U.S. Air Force Academy, USAF Academy, CO 80840-6400, United States
}

\section{A R T I C L E I N F O}

\section{Article history:}

Received 5 March 2012

Received in revised form 6 September 2012

Accepted 9 October 2012

Available online 7 November 2012

\section{Keywords:}

Reduced order modeling

Unsteady aerodynamics

Computational fluid dynamics

Dynamic stall vortex

\begin{abstract}
A B S T R A C T
The challenges in the modeling of the unsteady lift of a two-dimensional NACA 0012 airfoil oscillating and translating at large incidence angles are investigated in this paper. Forced oscillation motions with two reduced frequency values of 0.1 and 1.0 were used. The CFD results show that a hysteresis loop was developed in the lift variation with angle of attack; shapes of the loops change significantly with reduced frequency value. A dynamic stall vortex was identified in the pitching and plunging motions with $k=0.1$. This vortex delays the onset of flow separation over the upper surface to a higher incidence than would occur in steady conditions. The unsteady lift of the very fast motion is enhanced significantly due to the formation of a compression wave on the lower surface and an expansion wave on the upper surface. The results show clear limitations of unsteady aerodynamic theories for modeling motions at high incidences. Also, the CFD solution of indicial functions show a large oscillation at angles of attack beyond the stall angle, but the predictions of a model based on Radial Basis Function matches CFD values quite well.
\end{abstract}

(c) 2012 Elsevier Masson SAS. All rights reserved.

\section{Introduction}

Modern fighter aircraft often maneuver at angles of attack well beyond stall [48]. Having a reliable stall recovery control depends to a large extent on the accuracy of an aerodynamic model for these situations. High angle of attack aerodynamics is characterized by extensive unsteady flow separation with significant hysteresis loops in the aerodynamic coefficients due to dynamic stall phenomenon $[46,44,43]$. There are only limited theories available for the prediction of the unsteady aerodynamic loads. Lomax [39], for example, used the linearized Euler equations to derive exact initial values of the compressible indicial response of a flat plate airfoil to an impulse change in the angle of attack. Also, the unsteady lift of a two-dimensional wing impulsively moving from rest was studied numerically by Wagner [64] and experimentally by Walker [65]. Theodorsen [60], assuming potential flow, used conformal mapping to derive the solution of unsteady aerodynamic loads of a thin airfoil undergoing small pitching and plunging oscillations. However, these theories have not been validated for high angles of attack. There are also only limited experimental measurements available to determine the effects of unsteady flow on the aerodynamic forces and moments acting on an aircraft. This is mainly due to the complexity of unsteady flow and the limitations

\footnotetext{
* Corresponding author.

E-mail addresses: Mehdi.Ghoreyshi@usafa.edu (M. Ghoreyshi),

Russ.Cummings@usafa.edu (R.M. Cummings).

1 NRC Research Fellow.

2 Professor of Aeronautics.
}

of existing test facilities [23]. Also, flight tests are very expensive and take place late in the design cycle. With recent advances in computational methods, the computational modeling of flow around a maneuvering aircraft at high angles of attack are now possible. At a high fidelity level, a full-order mathematical model can be built based on the unsteady solution of the discretized Navier-Stokes equations $[17,16]$. This approach is computationally very expensive since such a model requires a large number of simulations at different motion amplitude and frequency values. An alternative is the development of a Reduced Order Model (ROM) of unsteady aerodynamic loads using CFD from an appropriate training maneuver(s). Although, the current available ROMs have been utilized for many applications, their application to the prediction of aerodynamic loads at high angles of attack could be a challenging task.

This paper attempts to investigate the challenges and issues of modeling the unsteady aerodynamics of high angles of attack using CFD. The test case is a two-dimensional airfoil undergoing relatively slow and fast oscillations and translations. The available unsteady aerodynamic theories are reviewed first, followed by a description of the flow solver. Three reduced order models are investigated in this work: indicial functions, Radial Basis Functions, and a state-space model. The mathematical models are presented and the way to identify model parameters from CFD is detailed. This includes response type CFD calculations, CFD maneuvering for training purposes, and extraction of flow separation points from CFD simulations. The solution of some rapid plunging and pitching motions are then presented. Finally, the limitations of each model to predict these solutions are discussed. 


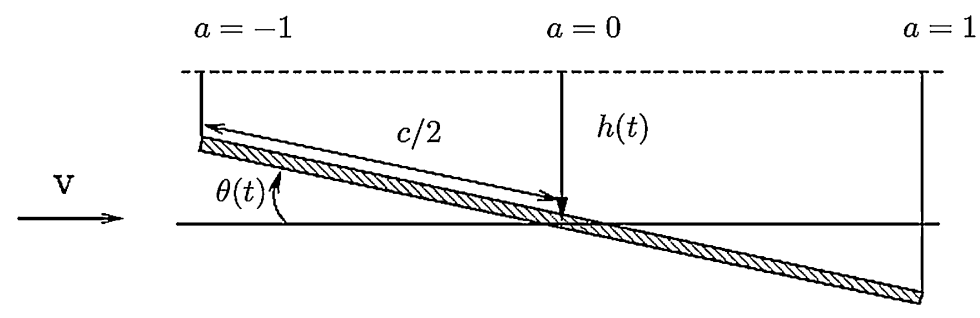

Fig. 1. Airfoil pitch and plunge maneuvers.

\section{Formulation}

\subsection{Unsteady aerodynamic theories}

An airfoil performing pitching and plunging motions is considered in this work. These motions are shown in Fig. 1 and described by:

$\theta(t)=\theta_{A} \cos \left(\omega_{\theta} t\right), \quad h(t)=H \cos \left(\omega_{h} t\right)$

where $\theta_{A}$ and $\omega_{\theta}$ are the pitch amplitude and rotational velocity, respectively, $h$ is the vertical placement of the airfoil, $H$ is the plunge amplitude, and $\omega_{h}$ denotes the oscillatory frequency. The reduced frequencies of $k_{\theta}$ and $k_{h}$ are defined as $\omega_{\theta} c / 2 V$ and $\omega_{h} c / 2 V$, where $c$ is the airfoil chord and $V$ is the free-stream velocity. The plunge motion has no rotation but the angle of attack changes due to the vertical displacements of the grid; this angle is named the "effective angle of attack", which is denoted by $\alpha_{e}$ and is defined as:

$\alpha_{e}=\tan ^{-1}\left(\frac{\dot{h}}{V}\right)$

The maximum effective angle of attack for a plunge starting at zero degrees angle of attack is determined by the Strouhal number defined as St $=2 f H / V$, such that $\alpha_{e}^{\max }=\tan ^{-1}(\pi \mathrm{St})$. The Strouhal number is a dimensionless number that is useful for describing unsteady flow of oscillating geometries [38]. There are a number of theories to predict the aerodynamic loads of pitching and plunging motions. Wagner [64] developed a theory based on potential flow, and detailed the unsteady lift coefficient of an airfoil undergoing a plunging motion as:

$C_{L}=2 \pi \int_{0}^{t} \Phi(t-\tau) \frac{d \alpha_{e}}{d t} d \tau$

where $C_{L}$ is the lift force coefficient and $\Phi(t)$ is the Wagner function and was approximated in non-dimensional time by a two-pole exponential function [35]

$\Phi(s)=1-0.165 \exp (-0.0455 s)-0.335 \exp (-0.3 s)$

where $s=2 \mathrm{Vt} / \mathrm{c}$ is a non-dimensional time. This function is shown graphically in Fig. 2(a). Wagner's function shows that the unsteady lift increases asymptotically in time to reach the quasi-steady value at one degree angle of attack, while it reaches almost $90 \%$ of this value after traveling a distance equivalent to 15 semi-chords [67]. Also, Lomax [39] used the linearized Euler equations to derive exact initial values of Wagner's function for a flat-plate airfoil

$\Theta(s)=\frac{1}{2 \pi} \frac{4}{M}\left(1-\frac{1-M}{2 M} s\right)$

where $M$ is the Mach number. Note that Lomax's function is valid only for the initial time of plunging motions. Theodorsen [60], assuming potential flow, used the method of conformal mapping to derive the solution of unsteady aerodynamic loads of a thin airfoil undergoing small pitching and plunging oscillations. His theory was expanded by Hodges and Pierce [26] for the lift and the pitch moment about the quarter-chord as

$$
\begin{aligned}
& C_{L}=2 \pi\left[\frac{\dot{h}}{V}+\theta+\frac{c \dot{\theta}}{2 V}\right] C(k)+\frac{\pi}{2}\left(\frac{c \ddot{h}}{V^{2}}+\frac{c \dot{\theta}}{V}-\frac{a c^{2} \ddot{\theta}}{V^{2}}\right) \\
& C_{m}=-\frac{\pi}{2}\left[\frac{1}{2} \frac{c \ddot{h}}{V^{2}}+\frac{\dot{c} \theta}{V}+\left(\frac{1}{8}-\frac{a}{2}\right) \frac{c^{2} \ddot{\theta}}{V^{2}}\right]
\end{aligned}
$$

where $a$ is the pitch axis location with respect to the half-chord as shown in Fig. 1. In this definition, the leading edge and trailing edge correspond to values of $a=-1$ and $a=1$, respectively. In Eq. (6), the effective angle of attack is:

$\alpha_{e}=C(k)\left[\frac{\dot{h}}{V}+\theta+\frac{c \dot{\theta}}{2 V}\right]$

where the first and second terms show the circulatory and noncirculatory effects, respectively. Non-circulatory effects, also called added mass, are generated from motion accelerations. Circulatory effects, on the other hand are generated from the differences in the velocity on the upper and lower surfaces of the airfoil. The airfoil pitch moment about the quarter-chord only has non-circulatory effects (the non-circulatory terms are usually negligible). The function $C(k)$ is called Theodorsen's function and is given as:

$C(k)=\frac{H_{1}^{(2)}(k)}{H_{1}^{(2)}(k)+i H_{0}^{(2)}(k)}$

where $H_{0}$ and $H_{1}$ are Hankel functions of the first and the second kind. The Theodorsen's function is real and equals 1 for the steady case (i.e. $k=0$ ) and includes an imaginary and a real part for moving airfoil. These parts are expressed as $C(k)=F(k)-i G(k)$ and shown in Fig. 2(b) for reduced frequencies up to 2 . The figure shows that $F(k)$ reduces with reduced frequency, thus the phase lag between the motion variables and unsteady loads is increased. The Theodorsen's function is related to the Wagner's function as [6]:

$C(k)=(i k) \int_{0}^{\infty} \Phi(\tau) e^{-i k \tau} d \tau$

and is approximated as

$C(k)=1-\frac{0.165(i k)}{i k+0.0455}-\frac{0.335(i k)}{i k+0.3}$

Also, Küssner [31] defined a "Wake Vortex Function", later named the Küssner function, $\Psi(t)$, to find the unsteady lift of an airfoil in response to an arbitrary gust with an upwash velocity of $\omega_{g}$ given as:

$C_{L}=2 \pi / V\left(\omega_{g}(0) \Psi(s)+\int_{0}^{s} \Psi(s-\sigma) \frac{d \omega_{g}(\sigma)}{d t} d \sigma\right)$ 


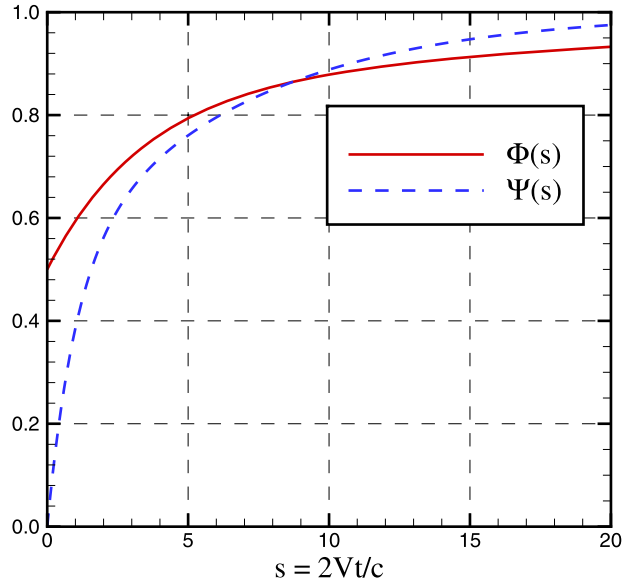

(a) Wagner and Küssner functions

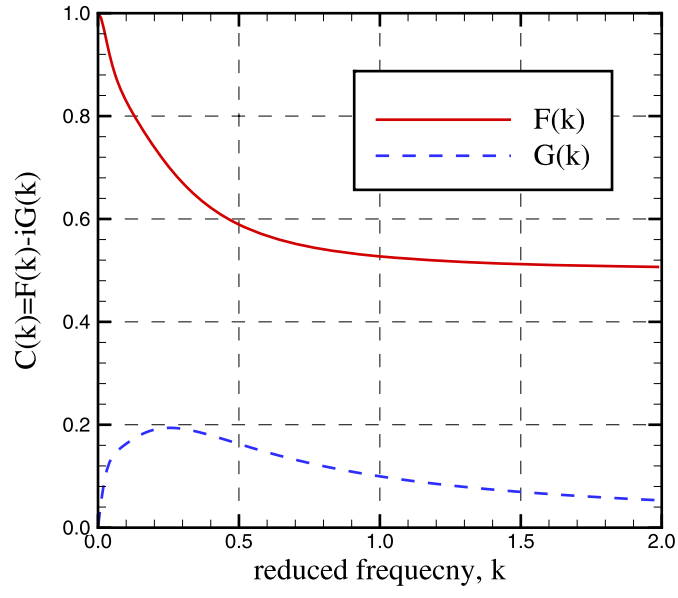

(b) Theodorsen's functions

Fig. 2. Unsteady aerodynamic force functions for incompressible flow over an airfoil.

where, $s=2 V t / c$ is the non-dimensional time. This function can be related to Wagner's function using a Fourier-integral and was approximated in terms of the Jones exponential expansion as:

$$
\Psi(s)=1-0.5 \exp (-0.15 s)-0.5 \exp (-s)
$$

Fig. 2(a) compares the Küssner and Wagner functions. The Küssner function is zero at $s=0$ and reaches unity for very large $s$ values. Note that these theories are limited only to linear and incompressible flows.

\subsection{Reduced-order aerodynamic models}

Recent efforts on the development of ROMs can be classified into two types: time domain or frequency domain approaches [40]. The frequency domain models are obtained from matching transfer functions computed from the measured input-output data [27]. Examples of the frequency domain ROMs are the indicial response method $[3,62,61]$ and a frequency-domain model based on Proper Orthogonal Decomposition (POD) $[25,66]$. The time domain models are based on the state space representation by matching time histories of measured data. Some examples of time domain ROMs include the unit sample response $[15,57,49,18]$, Volterra theory [56, 55,54,9], Radial Basis Functions (RBF) [29,19,7] and state-space modeling $[20,32]$. In this paper, the mathematical model of indicial functions, Radial Basis Functions, and state-space method are presented.

\subsubsection{Indicial functions}

The transient aerodynamic response due to a step change in a forcing parameter, such as angle of attack or pitch rate is a so-called "indicial function". Assuming that the indicial functions are known, the aerodynamic forces and moments induced in any maneuver can be estimated by using the well-known Duhamel superposition integral [36]. Tobak et al. [62,61] and Reisenthel et al. [50,51] detailed the superposition process for the modeling of unsteady lift and pitch moment from angle of attack and pitch rate indicial functions. If the time responses in lift due to the step changes in angle of attack, $\alpha$, and angular velocity, $q$, are known, then the total produced lift at time $t$ can be obtained as:

$$
\begin{aligned}
C_{L}(t)= & C_{L 0}+\frac{d}{d t}\left[\int_{0}^{t} C_{L \alpha}(t-\tau, \alpha) \alpha(\tau) d \tau\right] \\
& +\frac{d}{d t}\left[\int_{0}^{t} C_{L q}(t-\tau, \alpha) q(\tau) d \tau\right]
\end{aligned}
$$

where $C_{L 0}$ denotes the zero-angle of attack lift coefficient and is found from static calculations; $M$ is the free-stream Mach number; $C_{L \alpha}$ and $C_{L q}$ are lift indicial functions. Likewise, the pitch moment is estimated as follows:

$$
\begin{aligned}
C_{m}(t)= & C_{m 0}+\frac{d}{d t}\left[\int_{0}^{t} C_{m \alpha}(t-\tau, \alpha) \alpha(\tau) d \tau\right] \\
& +\frac{d}{d t}\left[\int_{0}^{t} C_{m q}(t-\tau, \alpha) q(\tau) d \tau\right]
\end{aligned}
$$

where $C_{m \alpha}$ and $C_{m q}$ are pitch moment indicial functions. The response functions due to pitch rate, i.e. $C_{L q}(\alpha)$ and $C_{m q}(\alpha)$ can be estimated using a time-dependent interpolation scheme from the observed responses. These values are next used to estimate the second integrals in Eqs. (14) and (15), however, the estimation of the integrals with respect to the angle of attack needs more explanation. Assuming a set of angle of attack samples of $\alpha=\left[\alpha_{1}, \alpha_{2}, \ldots, \alpha_{n}\right]$, the lift and pitch moment responses to each angle of $\alpha_{i}, i=1,2, \ldots, n$ are denoted as $A_{j_{\alpha}}\left(t, \alpha_{i}\right)$, where $j=$ $[L, m]$ representing lift and pitch moment respectively. In these response simulations, $\alpha(t)=0$ at $t=0$ and is held constant at $\alpha_{i}$ for all $t>0$. For a new angle of $\alpha^{*}>0$, the responses of $A_{j_{\alpha}}\left(t, \alpha_{k}\right)$ are being interpolated at $\alpha_{k}=\left[\alpha_{1}, \alpha_{2}, \ldots, \alpha_{s}\right]$, such that $0<\alpha_{1}<\alpha_{2}<\cdots<\alpha_{s}$ and $\alpha_{s}=\alpha^{*}$. These angles can have a uniform or non-uniform spacing. The indicial functions of $C_{j \alpha_{k}}$ for $k=1, \ldots, s$ at each interval of $\left[\alpha_{k-1}, \alpha_{k}\right]$ are defined as

$$
\begin{aligned}
C_{j \alpha_{1}} & =\frac{A_{j_{\alpha}}\left(t, \alpha_{1}\right)-C_{j 0}}{\alpha_{1}} \\
C_{j \alpha_{k}} & =\frac{A_{j_{\alpha}}\left(t, \alpha_{k}\right)-A_{j_{\alpha}}\left(t, \alpha_{k-1}\right)}{\alpha_{k}-\alpha_{k-1}}
\end{aligned}
$$

where $C_{j 0}$ denotes the zero angle of attack lift or pitch moment coefficient. The interval indicial functions are then used to estimate the values of first integrals in Eqs. (14) and (15). These steps can easily be followed for a negative angle of attack, i.e. $\alpha^{*}<0$. Once this model is created, the aerodynamic response to a wide range of motions can be predicted on the order of a few seconds of computational time. However, the above model still requires determination of indicial functions.

Experimental tests are practically nonexistent for indicial function measurements due to wind tunnel constraints. Limited analytical expressions of indicial functions exist for two-dimensional 

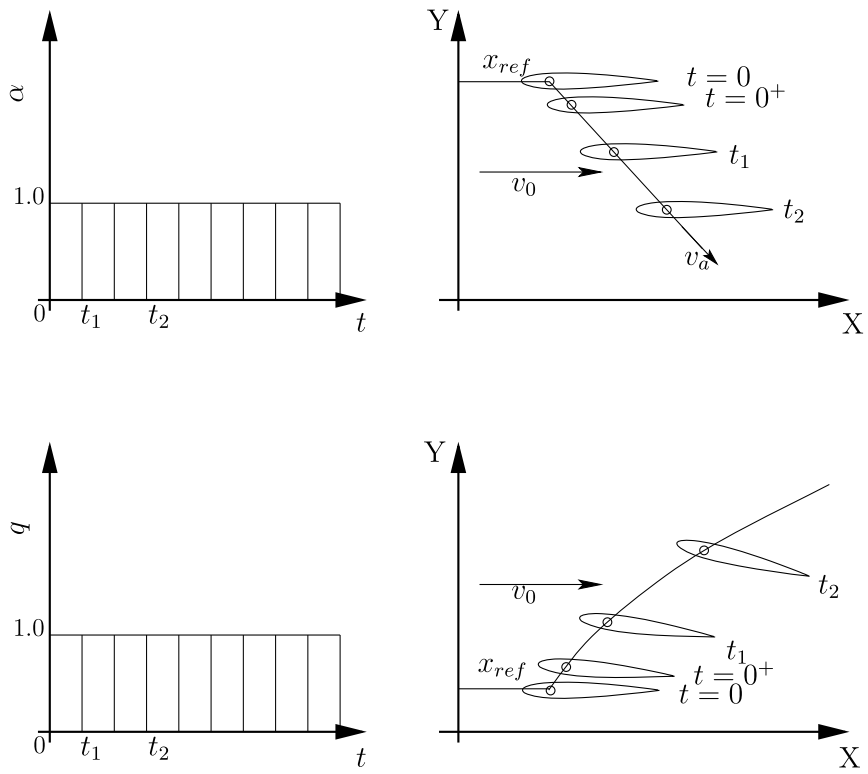

Fig. 3. The grid motion for modeling a step change in angle of attack and pitch rate.

airfoils [41]. However, these analytical expressions are not valid for aircraft configurations due to the three-dimensional tip vortices. An alternative is to use CFD, but special considerations are required to simulate step responses in CFD. Singh and Baeder [57] used a surface transpiration approach to directly calculate the angle of attack indicial response using CFD. Ghoreyshi et al. [18] also described an approach based on a grid motion technique for CFD-type calculation of linear and nonlinear indicial functions with respect to angle of attack and pitch rate.

In this work, the indicial functions are directly calculated from URANS simulations and using a grid motion tool. Cobalt, the flow solver used, uses an arbitrary Lagrangian-Eulerian formulation and hence allows all translational and rotational degrees of freedom [18]. The code can simulate both free and specified six degree of freedom (6DoF) motions. The rigid motion is specified from a motion input file. For the rigid motion the location of a reference point on the aircraft is specified at each time step. In addition the rotation of the aircraft about this reference point is also defined using the rotation angles of yaw, pitch and roll (bank). The aircraft reference point velocity, $V_{a}$, in an inertial frame is then calculated to achieve the required angles of attack and side-slip, and the forward speed. The velocity is then used to calculate the location. The initial aircraft velocity, $V_{0}$, is specified in terms of Mach number, angle of attack and side-slip angle in the main file. The instantaneous aircraft location for the motion file is then defined from the relative velocity vector, $V_{a}-V_{0}$. For CFD-type calculation of a step change in angle of attack, the grid immediately starts to move at $t=0$ to the right and downward as shown in Fig. 3. The translation continues over time with a constant velocity vector. Since there is no rotation, all the effects in aerodynamic loads are from changes in the angle of attack. For a unit step change in pitch rate, the grid moves and rotates simultaneously. The grid starts to rotate with a unit pitch rate at $t=0$. To hold the angle of attack zero during the rotation, the grid moves right and upward in Fig. 3. All indicial function computations started from a steady-state solution and then advanced in time using second-order accuracy with five Newton sub-iterations. The steady state solutions correspond to zero degrees angle of attack and side-slip for the Mach number of interest.

\subsubsection{Radial basis functions}

The nonlinear and unsteady aerodynamics can be viewed as a multi-input/multi-output dynamic system with a mathematical model in state space given by an ordinary differential equation

$\dot{x}_{t}=f\left(x_{t}, u_{t}\right)$

and an output equation as:

$y_{t}=h\left(x_{t}\right)$

with given initial condition $x=x_{0}$, for $t=t_{0}$ and

$x_{t} \in \mathbb{R}^{n}, \quad y_{t} \in \mathbb{R}^{m}, \quad u_{t} \in \mathbb{R}^{r}$

where $x$ is a $n$-dimensional vector of internal state variables over the field $\mathbb{R}$ of real numbers, $u$ is a vector of the inputs to system, and $y$ is $m$-dimensional vector of system outputs. For pitching and plunging motions considered in this work, $\mathbf{u}$ takes the form of

$\mathbf{u}(t)=(\alpha(t), \dot{\alpha}(t), \ddot{\alpha}(t))$

For a discrete-time dynamical systems, the equations change to

$x_{\mathrm{k}+1}=f\left(x_{\mathrm{k}}, u_{\mathrm{k}}\right)$

$y_{\mathrm{k}}=h\left(x_{\mathrm{k}}\right)$ for $\mathrm{k}=0,1, \ldots$

where $\mathrm{k}$ is an integer value showing discrete time values. The state function $f$ is a smooth function that maps the current state $x_{\mathrm{k}}$ and the input $u_{\mathrm{k}}$ into a new state $x_{\mathrm{k}+1}$, and the output function $h$ maps the state $x_{\mathrm{k}}$ into the output $y_{\mathrm{k}}$ given as [2]

$f: \mathbb{R}^{n} \times \mathbb{R}^{r} \mapsto \mathbb{R}^{n}, \quad h: \mathbb{R}^{n} \mapsto \mathbb{R}^{m}$

In this system, the outputs can be determined from the states at time instant $\mathrm{k}$ only, therefore the past history of the system is irrelevant [1]. If the state variables are directly measured, the functions $f$ and $h$ can be approximated using neural networks or surrogate-based models. However, in many practical situations, measuring all state variables is limited. Referring to unsteady aerodynamic problem, the discretized governing equations of fluid dynamics serve as the state space functions with an internal state vector of $(\rho, p, u, v, w, E)$ that corresponds to the values of density, pressure, velocity components, and energy at each grid point. This large amount of data makes the identification of Eq. (21) a very complex task. Fortunately, there are available methods that allows us to reconstruct the state space model by mapping only the input and output data.

For a finite-time interval and a system described by Eq. (21), Levin and Narendra [37] used the Implicit Function theorem [30] to write the output vector at any instant as a function of the past $n$ values of the inputs and the past $n$ values of the outputs, i.e.:

$y_{\mathrm{k}+1}=\Psi\left(y_{\mathrm{k}}, y_{\mathrm{k}-1}, \ldots, y_{\mathrm{k}-n+1}, u_{\mathrm{k}}, u_{\mathrm{k}-1}, \ldots, u_{\mathrm{k}-n+1}\right)$

In Eq. (22), $\Psi$ is a vector-valued nonlinear function that maps the inputs to the outputs, and $n$ is an integer representing the past values in the output and input. Eq. (22) preserves the characteristics of the state-space model but no longer depends on system internal states. The input-output mapping, $\Psi$, can be learned using neural networks when input and output time histories are available. This network is often named a recurrent neural network [1], where the network output becomes part of the next input vector [11]. For the pitching airfoil, the lift and pitch moment can be expressed as function of three parameters of $\alpha_{0}, \alpha_{A}$, and $k$, which denote the mean angle of attack, the pitch amplitude, and the pitch reduced frequency, respectively. These independent variables form a parameter space, which represents the envelope of all possible flow conditions in which the aircraft configuration is 


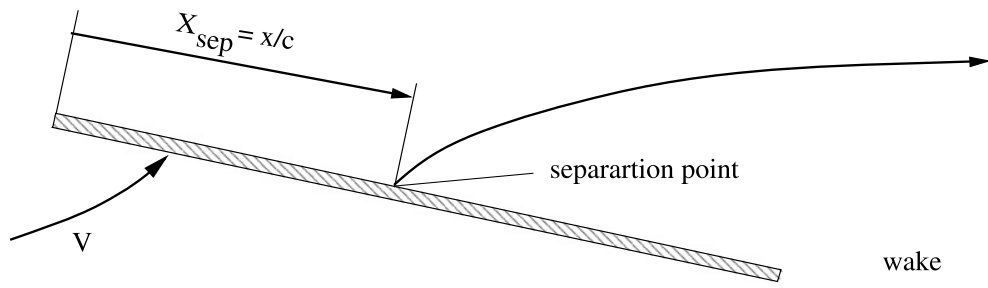

Fig. 4. The trailing edge separation point.

expected to operate. To generate a consistent set of unsteady aerodynamic loads in response to a given aircraft motion time history, the training cases at which CFD solutions are calculated should be representative of the expected flow conditions. In this paper function $\Psi$ is approximated with Radial Basis Functions.

Given an input vector of $\left\{X_{j}^{c}: j=1, \ldots, p\right\}, X_{j}^{c} \in \mathbb{R}$ and a corresponding output vector of $\left\{Y_{j}^{c}: j=1, \ldots, p\right\}, Y_{j}^{c} \in \mathbb{R}$, the RBF approximates the output at a new given point as:

$\hat{Y}\left(X^{*}\right)=\sum_{\mathrm{k}=1}^{P} \alpha_{\mathrm{k}} \Phi_{i}(X)$

such that

$\hat{Y}\left(X_{j}^{c}\right)=Y_{j}^{c}, \quad$ for $j=1,2, \ldots, p$

where $\alpha_{\mathrm{k}}$ are the weights of the linear combiners and the functions $\Phi_{i}$ are named Radial Basis Functions and often described by a Gaussian basis function as

$\Phi_{i}(X)=\exp \left(-\frac{\left\|X^{*}-X_{j}^{c}\right\|^{2}}{\beta^{2}}\right)$

in above $\beta$ is a real variable to be decided by user, $\|$.$\| denotes$ the Euclidean norm such that the functions $\Phi_{i}$ will vanish at sufficiently large values of $\left\|X^{*}-X_{j}^{c}\right\|$. The remaining problem is how to choose $n$ such that the reconstructed model accurately represents the state-space model described by Eq. (22). The reconstructed model exactly matches Eq. (21) in a neighborhood of the equilibrium state by setting $n$ at most to $2 r+1$, where $r$ is the input vector dimension [10]. In this paper the value of $n$ is selected using "trial and error" attempts.

\subsubsection{State space modeling}

A state space model detailed by Goman and Khrabov [21] is considered in this work. In this approach, the unsteady aerodynamic loads are found from an input-output dynamical system in the form of:

$\frac{d x}{d t}=f(x, u)$

$C_{j}=g(x, u)$

where, $\mathbf{u}$ shows the system input vector and defined in Eq. (20), $C_{j}$ denote aerodynamic forces that for pitching and plunging motions are $j=L, D, m . x$ is a non-dimensional coordinate which describes the flow separation point on the upper surface of airfoil as shown in Fig. 4, such that $x=1$ refers to the attached flow, and $x=0$ means flow separation point is at the leading edge. In this paper, the lift coefficient of an airfoil is considered which its dependency on $\alpha$ and $x$ was approximated by Kichgof's model [20] based on constant pressure and linear cavitation theory:

$C_{L}^{n l}(\alpha, x)=(2 \pi) \sin \alpha\left(\frac{1+\sqrt{x}}{2}\right)^{2}$ where estimates the quasi-steady nonlinear lift due to the flow separation. For a pitching and plunging motion the total lift is then estimated as:

$C_{L}=C_{L}^{n l}(\alpha, x)+C_{L q}^{a t t} \frac{q c}{V}+C_{L \dot{\alpha}}^{a t t} \frac{\dot{\alpha} c}{V}$

where $C_{L q}^{a t t}$ and $C_{L \dot{\alpha}}^{a t t}$ are the constant aerodynamic derivatives estimated for the attached flow. These derivatives can be estimated using the methods as detailed by Da Ronch et al. [8]. Goman, and Khrabov [20] also rearranged Eq. (26) as:

$\tau_{1} \frac{d x(t)}{d t}+x(t)=x_{0}\left(\alpha-\tau_{2} \dot{\alpha}\right)$

where, $\tau_{1}$, and $\tau_{2}$ are time constants and determined from some training motions and $x_{0}$ shows the steady separation point and is available from steady simulations. Goman and Khrabov [21] approximated the time constants as $\tau_{1}=15 \mathrm{c} / \mathrm{V}$ and $\tau_{2}=0.5 \mathrm{c} / \mathrm{V}$, where $c$ is the airfoil chord and $V$ is the free stream velocity. A key aspect of this reduced order model is the extraction of separation point from CFD solutions. Next, more details of the flow solver and extraction of separation points are provided.

\subsection{CFD solver}

The flow solver used for this study is the Cobalt code [58] that solves the unsteady, three-dimensional and compressible NavierStokes equations in an inertial reference frame. These equations in integral form are:

$$
\begin{aligned}
& \frac{\partial}{\partial t} \iiint \mathbf{Q} d V+\iint(\hat{\mathbf{f}} \hat{i}+\mathbf{g} \hat{j}+\mathbf{h} \hat{k}) \cdot \hat{n} d S \\
& \quad=\iint(\mathbf{r} \hat{i}+\mathbf{s} \hat{j}+\mathbf{t} \hat{k}) \cdot \hat{n} d S
\end{aligned}
$$

where $V$ is the fluid element volume; $S$ is the fluid element surface area; $\hat{n}$ is the unit normal to $S ; \hat{i}, \hat{j}$, and $\hat{k}$ are the Cartesian unit vectors; $\mathbf{Q}=(\rho, \rho u, \rho v, \rho w, \rho e)^{T}$ is the vector of conserved variables, where $\rho$ represents air density, $u, v, w$ are velocity components and $e$ is the specific energy per unit volume. The vectors of $\mathbf{f}, \mathbf{g}$, and $\mathbf{h}$ represent the inviscid components and are detailed below

$$
\begin{aligned}
& \mathbf{f}=\left(\rho u, \rho u^{2}+p, \rho u v, \rho u w, u(\rho e+p)\right)^{T} \\
& \mathbf{g}=\left(\rho v, \rho v^{2}+p, \rho v u, \rho v w, v(\rho e+p)\right)^{T} \\
& \mathbf{h}=\left(\rho w, \rho w^{2}+p, \rho w u, \rho w v, w(\rho e+p)\right)^{T}
\end{aligned}
$$

where the superscript $T$ denotes the transpose operation. The vectors of $\mathbf{r}, \mathbf{s}$, and $\mathbf{t}$ represent the viscous components which are described as

$$
\begin{aligned}
& \mathbf{r}=\left(0, \tau_{x x}, \tau_{x y}, \tau_{x z}, u \tau_{x x}+v \tau_{x y}+w \tau_{x z}+k T_{x}\right)^{T} \\
& \mathbf{s}=\left(0, \tau_{x y}, \tau_{y y}, \tau_{y z}, u \tau_{x y}+v \tau_{y y}+w \tau_{y z}+k T_{y}\right)^{T} \\
& \mathbf{t}=\left(0, \tau_{x z}, \tau_{z y}, \tau_{z z}, u \tau_{x z}+v \tau_{z y}+w \tau_{z z}+k T_{z}\right)^{T}
\end{aligned}
$$




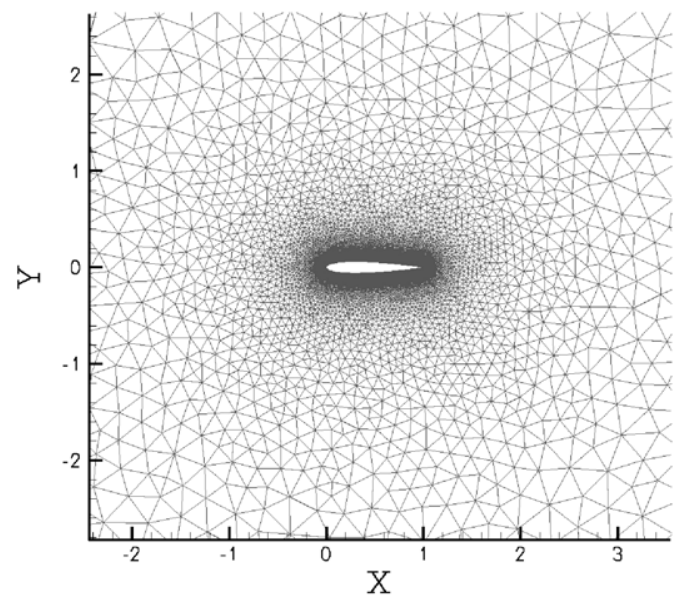

(a) NACA 0012 global grid view

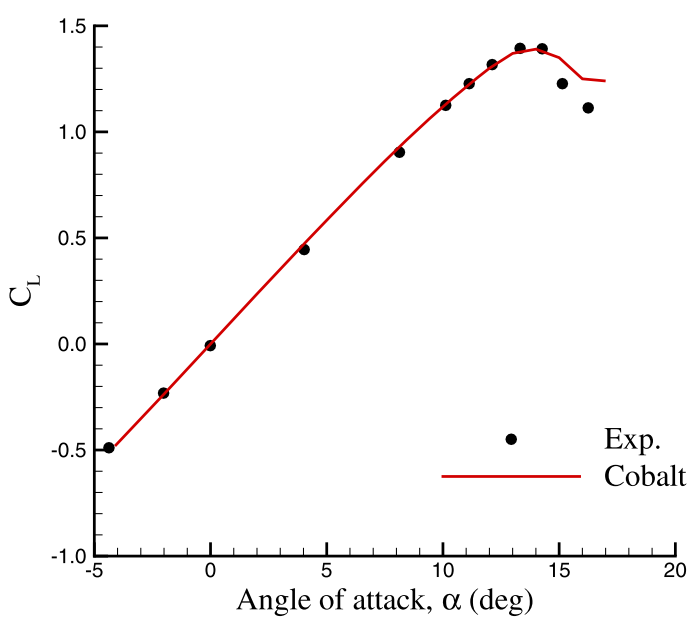

(c) Static lift coefficient

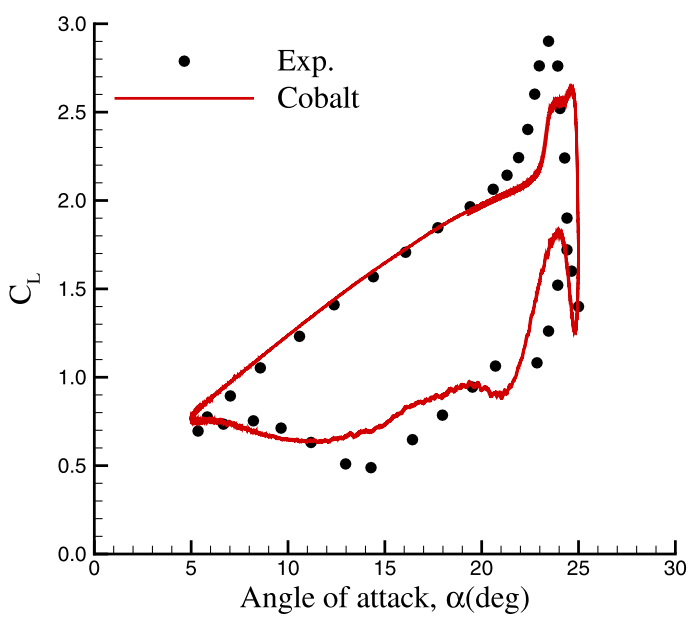

(e) $\alpha=15^{\circ}+10^{\circ} \sin (\omega t)$

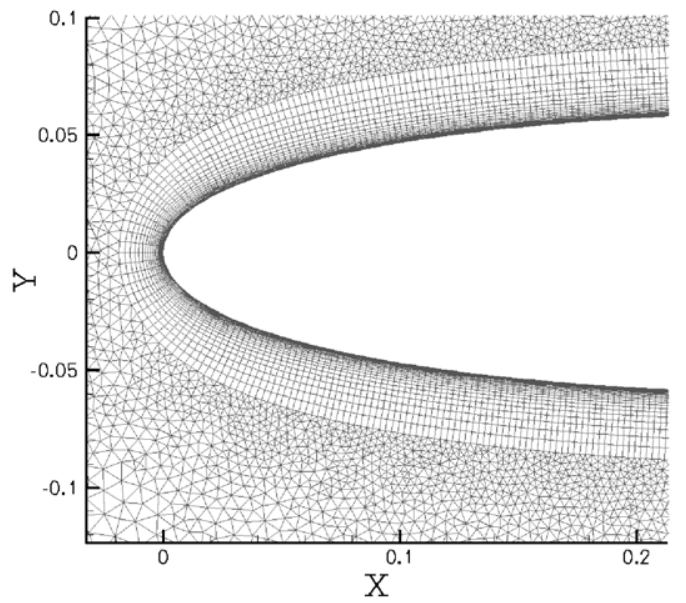

(b) NACA 0012 viscous layer

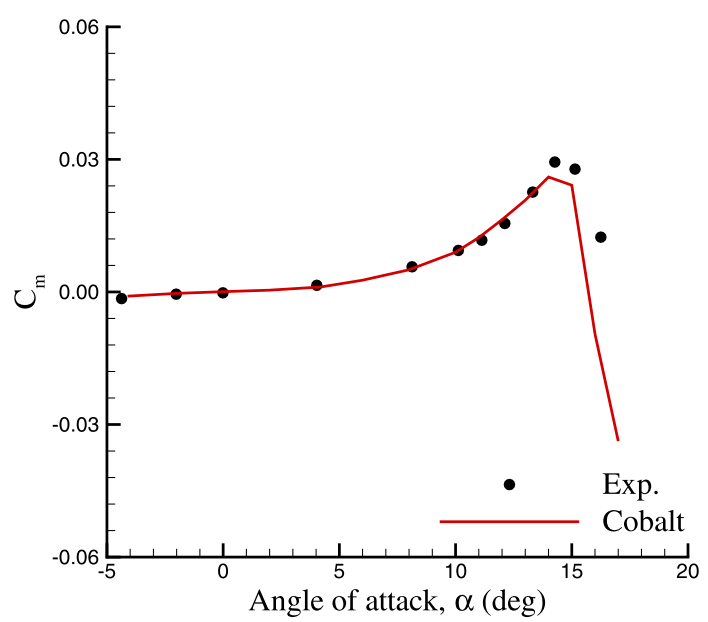

(d) Static pitch moment coefficient

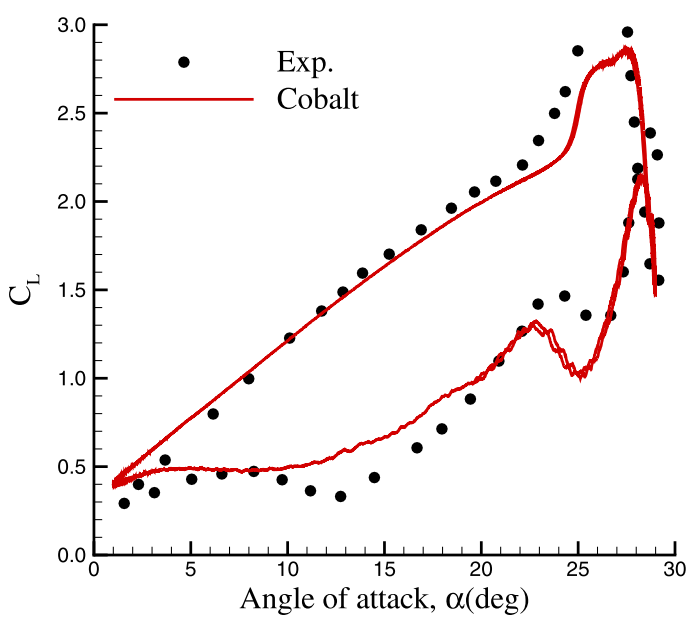

(f) $\alpha=15^{\circ}+14^{\circ} \sin (\omega t)$

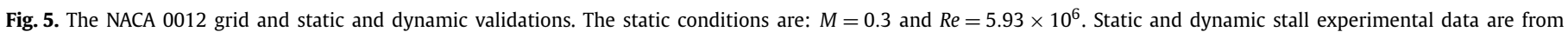
Ladson [33] and McAlister et al. [42], respectively. In dynamic stall experiments: $k=\omega c / 2 V=0.1$ and $M=0.09$.

where $\tau_{i j}$ are the viscous stress tensor components, $T$ is the temperature, and $k$ is the thermal conductivity. The ideal gas law and Sutherland's law closes the system of equations and the entire equation set is non-dimensionalized by free stream density and speed of sound [58]. The Navier-Stokes equations are discretized on arbitrary grid topologies using a cell-centered finite volume method. Second-order accuracy in space is achieved using the exact Riemann solver of Gottlieb and Groth [22], and least squares gradient calculations using $\mathrm{QR}$ factorization. To accelerate the solution of discretized system, a point-implicit method using analytic first-order inviscid and viscous Jacobians. A Newtonian sub-iteration method is used to improve time accuracy of the 


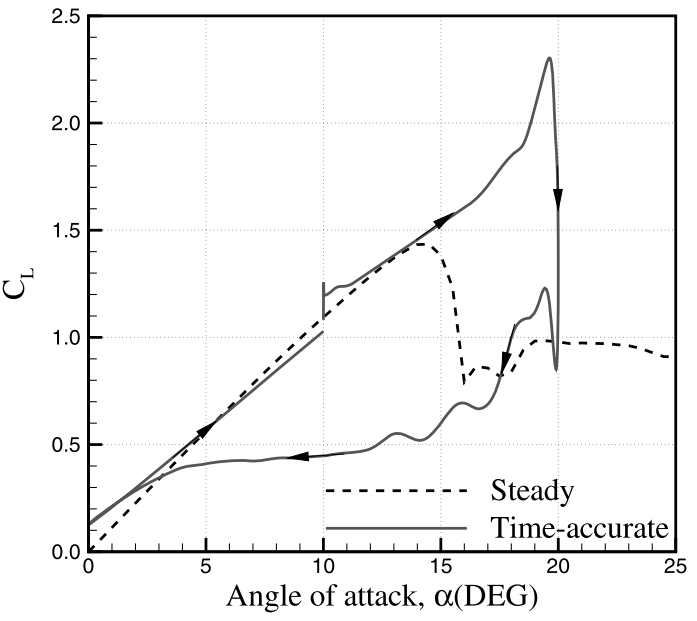

(a) Lift coefficient $-k=0.1$

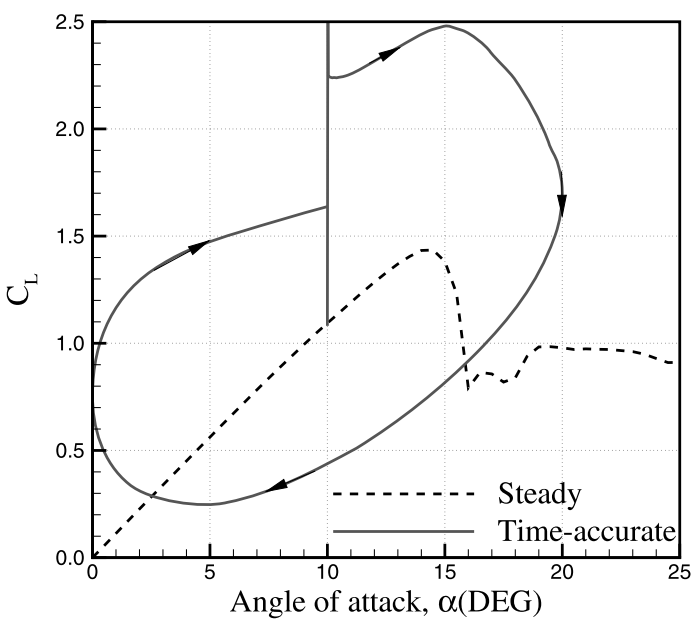

(c) Lift coefficient $-k=1.0$

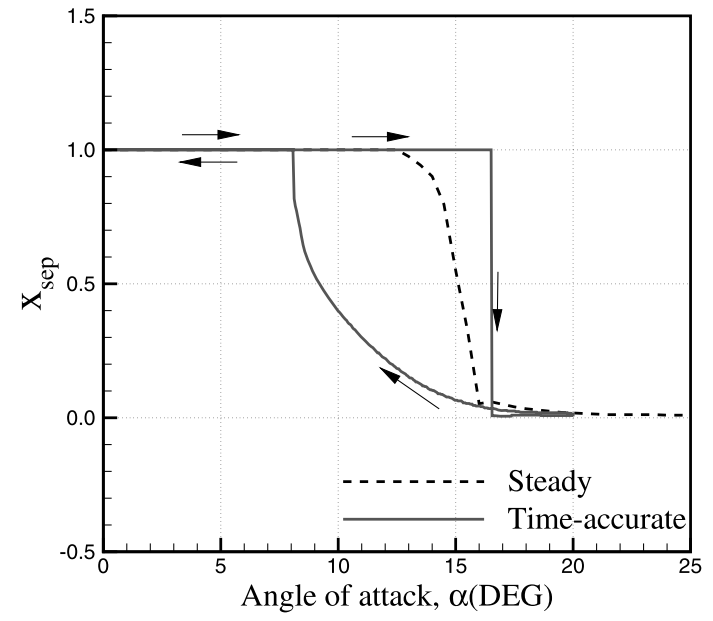

(b) Separation points $-k=0.1$

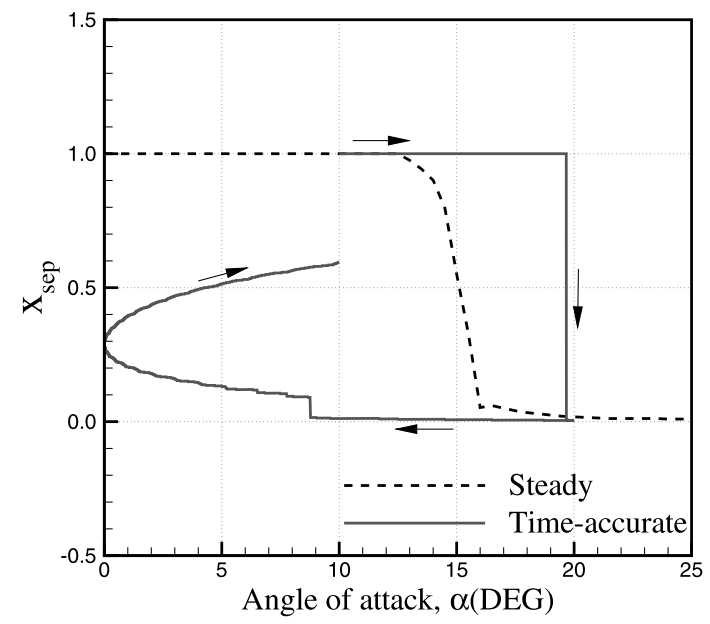

(d) Separation points $-k=1.0$

Fig. 6. Pitching airfoil: $M=0.3$.

point-implicit method. Tomaro et al. [63] converted the code from explicit to implicit, enabling Courant-Friedrichs-Lewy numbers as high as $10^{6}$. The Cobalt solver has been used at the Air Force Seek Eagle Office (AFSEO) and the United States Air Force Academy (USAFA) for a variety of unsteady nonlinear aerodynamic problems of maneuvering aircraft $[13,12,45,14,28]$.

\subsection{Extraction of separation points}

The accuracy of the state space method depends to a large extent on accurate identification of separation points from CFD simulations. For a two-dimensional airfoil, separation flows with adverse pressure gradients (retarded flows) give rise to a vanished wall shear stress at the point of separation [53]. If the adverse pressure gradients is not strong enough, the flow reattaches to the surface and a bounded area with backflow is formed, named separation bubble. For an airfoil oscillating and translating at high incidences, separation bubbles form, grow, and break up in time. A systematic approach is needed for identification of separation and re-attachment points. Surana et al. [59] detailed flow separation and attachment criteria:

$\mathbf{p}$ is a separation point if, and only if,

$\tau_{w}(\mathbf{p})=0, \quad \nabla_{x} \cdot \tau_{w}(\mathbf{p})<0, \quad \operatorname{det} \nabla_{x} \tau_{w}(\mathbf{p})>0$

where, $\tau_{w}$ shows the wall shear stress. Likewise, $\mathbf{p}$ is an attachment point if, and only if,

$$
\tau_{w}(\mathbf{p})=0, \quad \nabla_{x} \cdot \tau_{w}(\mathbf{p})>0, \quad \operatorname{det} \nabla_{x} \tau_{w}(\mathbf{p})>0
$$

Cobalt solver writes the wall shear stress on the upper surface of moving airfoil. The gradient, divergence, and curl of the shear stress vector are then calculated. The separation, and reattachment points are identified using Eqs. (34)-(35). For separation bubbles with a size larger than $0.1 c$, the $x$ term in Eq. (28) is equivalent to identified separation point. If no separation found, or separation bubbles be small, $x=1$.

\section{Test case}

The pitching and plunging simulations of NACA 0012 airfoil using Cobalt are considered. The computational mesh is rectangular with the airfoil geometry centrally located. The overview of the fine mesh is shown in Figs. 5(a)-(b). The minimal distance from the body to each of the outer boundaries is $20 c$. The no-slip, adiabatic wall boundary conditions is employed at the body surface and the modified Riemann-invariant condition was implemented at the far-field boundary. The mesh consists of prisms and tetrahedra and was generated using SolidMesh 2D. Jirásek et al. [29] performed the sensitivity study of the grid size and time step. The pitch axis and the moment reference point are set to $0.25 \mathrm{c}$ for all the results.

Experimental data for the steady and unsteady aerodynamics of the NACA 0012 airfoil are available. For steady simulations, the simulation conditions correspond to $M_{\infty}=0.3$ and 

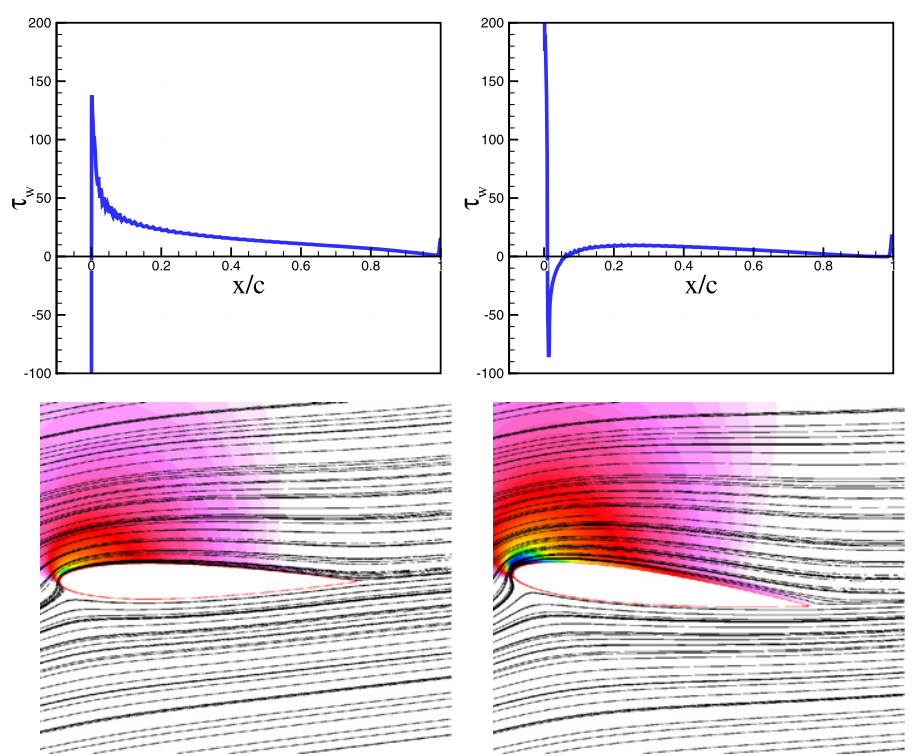

(a) $\alpha=10^{\circ}$
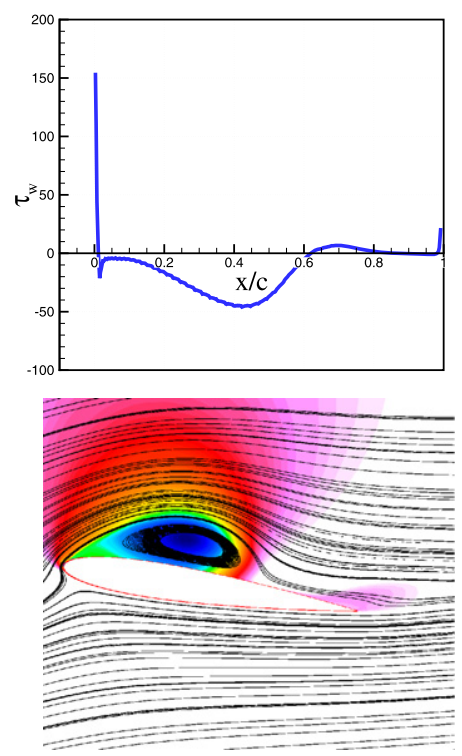

(e) $\alpha=18.6^{\circ}$

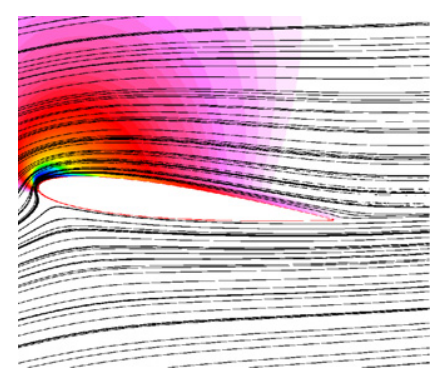

(b) $\alpha=16.2^{\circ}$

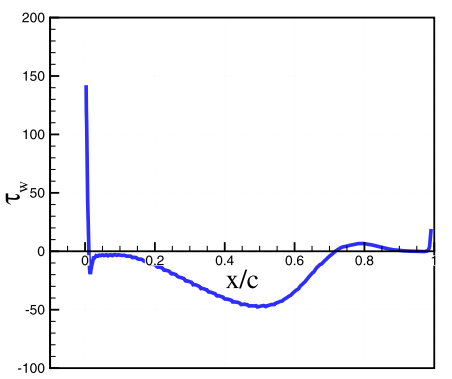

(f) $\alpha=18.9^{\circ}$

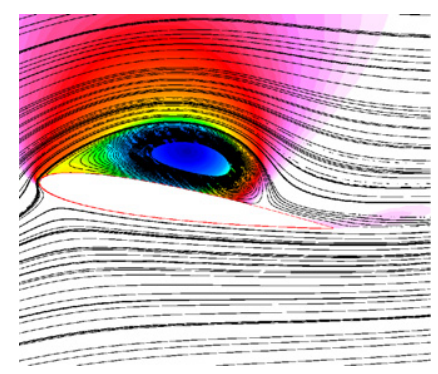

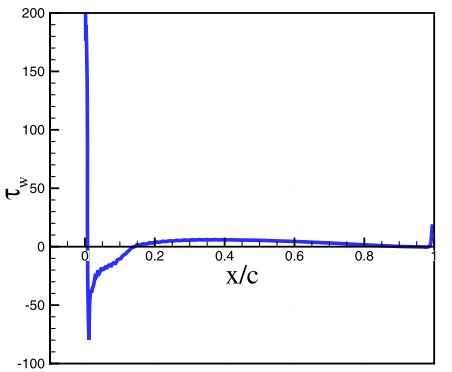

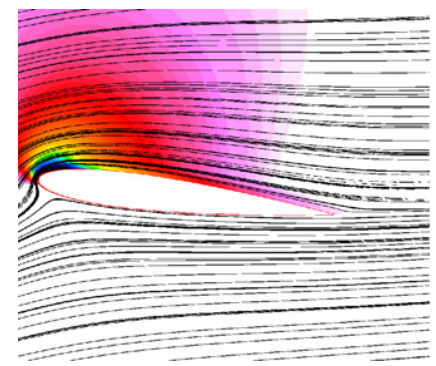

(c) $\alpha=16.7^{\circ}$
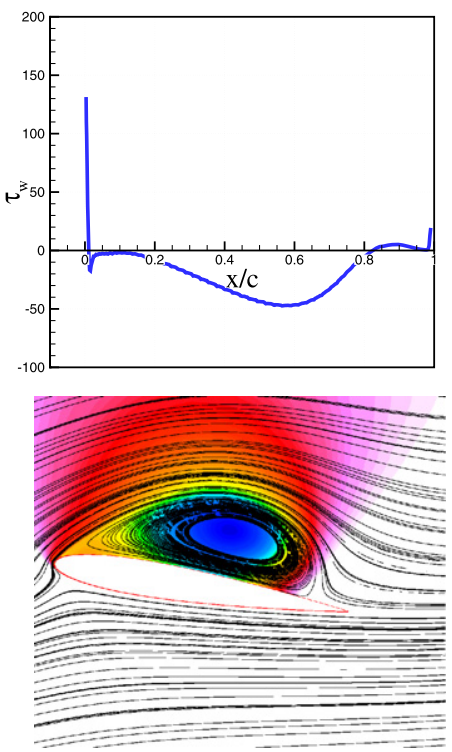

(g) $\alpha=19.2^{\circ}$
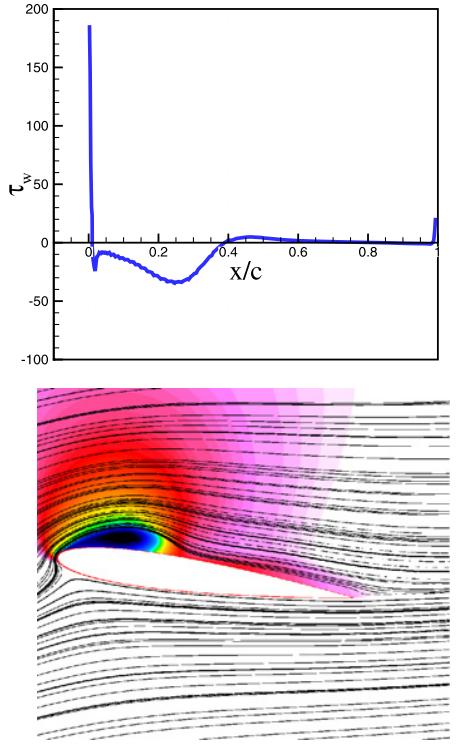

(d) $\alpha=17.7^{\circ}$
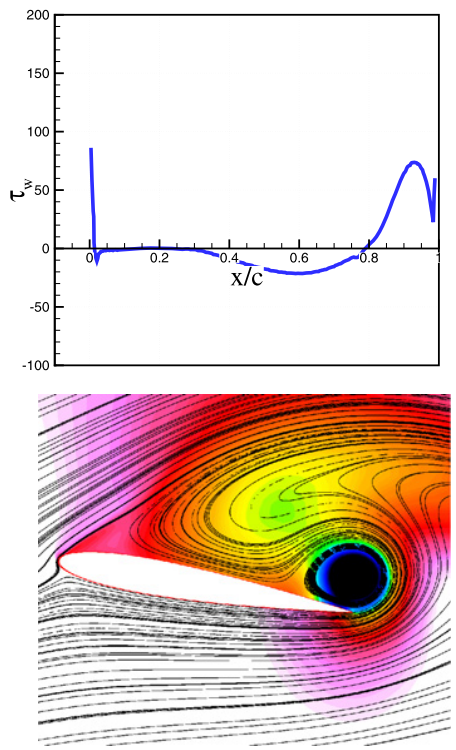

(h) $\alpha=20.0^{\circ}$

Fig. 7. Pitching-up airfoil: $M=0.3$ and $k=0.1$

$R e=8.93 \times 10^{6}$. All simulations were performed using the SpalartAllmaras (SA) turbulence model. The predictions of lift and pitch moment coefficients are compared with experimental data from Ladson [33] in Figs. 5(c)-(d). A very good agreement is obtained for the angles below 15 degrees. Also, the SA model accurately predicts the maximum lift, and the stall trend of experimental data.

Two pitch oscillation motions were selected from dynamic stall experiments of NACA 0012 airfoil [42]. The airfoil used in experiment is a simple geometry without modification and end plates. The motions are defined as $\alpha=15^{\circ}+10^{\circ} \sin (\omega t)$ and $\alpha=15^{\circ}+$ $14^{\circ} \sin (\omega t)$ with a reduced frequency of $k=0.1$ and Mach number of $M=0.09$. The CFD predictions of these motions are shown against experimental data in Figs. 5(e)-(f) which show the experimental data have very nonlinear hysteresis loops in the lift variation with angle of attack. Even for these unsteady cases at high incidence angles, URANS predictions show a good agreement, although Detached-Eddy Simulation (DES) flow modeling could help to capture the unsteady flows at such high angles of attack more accurately. For aerodynamics modeling work, the URANS predictions are considered reasonable simplifications and used in this work.

\section{Results}

The results of relatively slow and fast pitching and plunging NACA 0012 airfoil at $M=0.3$ are presented in this work. Pitch motions are defined as $\alpha=10^{\circ}+10^{\circ} \sin (w t)$. The translation motions are set in motion from an angle of attack of $10^{\circ}$ with a displacement amplitude that sets maximum effective angle to $20^{\circ}$. Both pitching and plunging are oscillation-type motions with reduced frequencies $(k=\omega c / 2 V)$ of 0.1 and 1.0. Figs. $6(\mathrm{a})-(\mathrm{c})$ present the lift coefficient variation with angle of attack in a pitching motion with $k=0.1$ and $k=1.0$, respectively. Also, the dashed curve shows the steady predictions. Figs. $6(\mathrm{a})-(\mathrm{c})$ show that significantly different hysteresis loops are found with respect to reduced frequency. For the pitching with $k=0.1$, the maximum unsteady lift coefficient is roughly twice as the maximum lift coefficient 

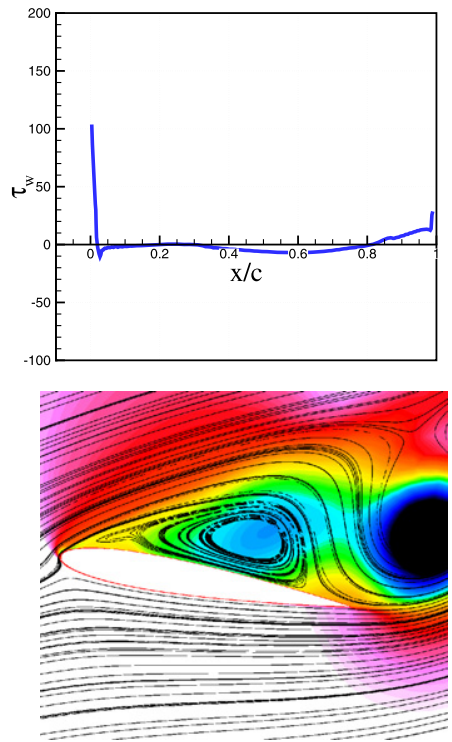

(a) $\alpha=19.8^{\circ}$
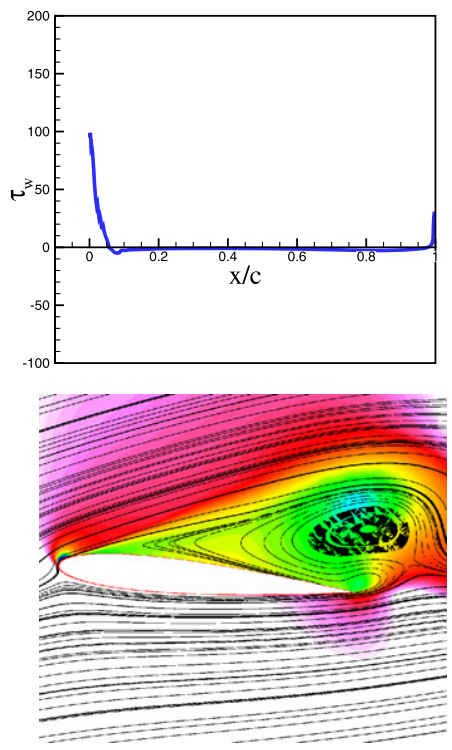

(e) $\alpha=15.3^{\circ}$
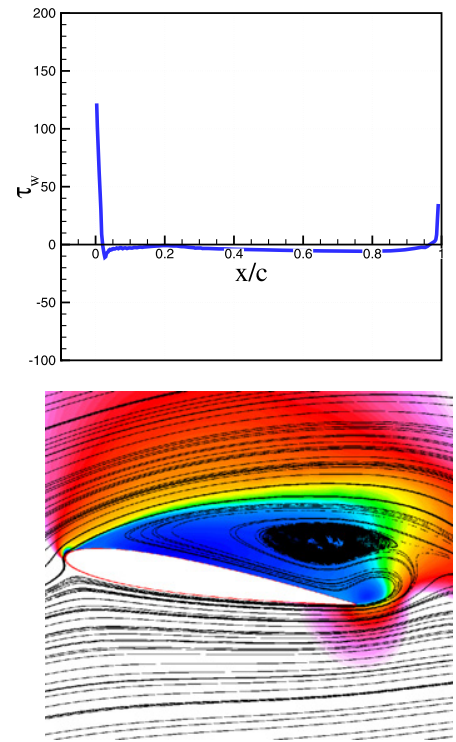

(b) $\alpha=19.1^{\circ}$
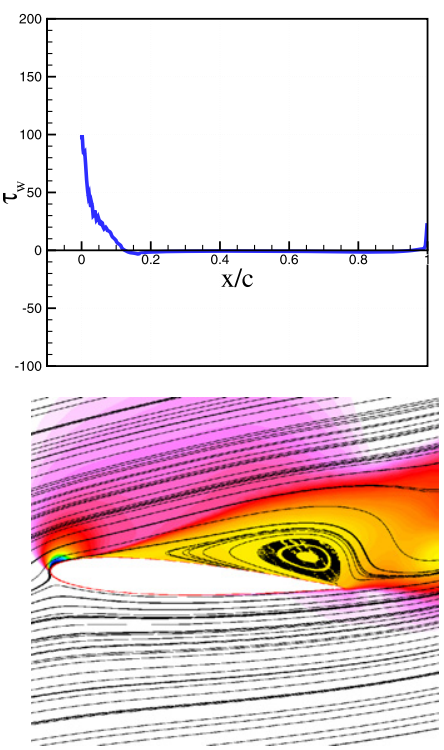

(f) $\alpha=13.5^{\circ}$
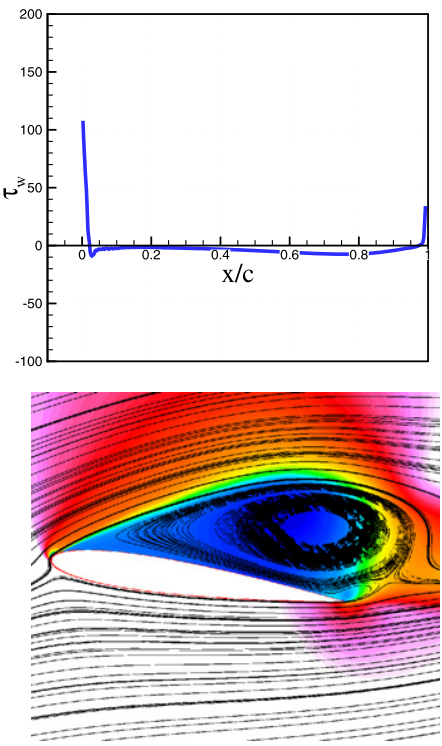

(c) $\alpha=18.2^{\circ}$
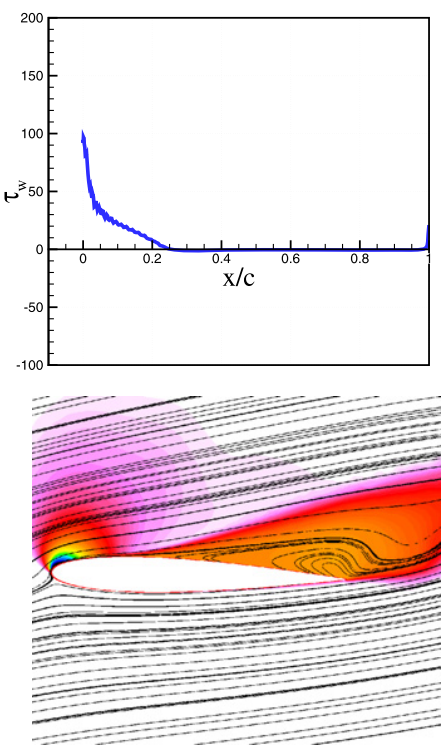

(g) $\alpha=11.5^{\circ}$
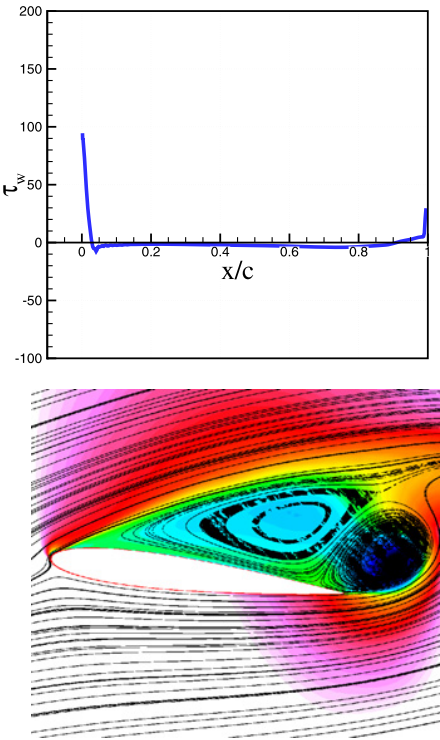

(d) $\alpha=16.9^{\circ}$
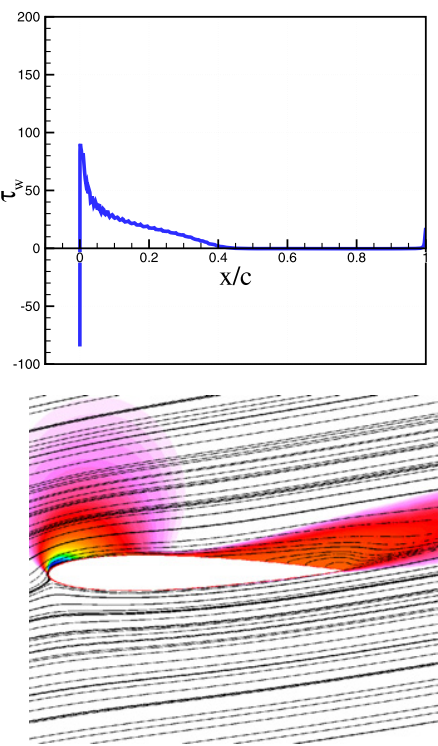

(h) $\alpha=9.5^{\circ}$

Fig. 8. Pitching-down airfoil: $M=0.3$ and $k=0.1$.

from the steady predictions. The instantaneous streamlines and upper surface wall shear stress during the upstroke are shown in Fig. 7. The visualization of flow solution shows no sign of flow separation for angles of attack up to $16^{\circ}$. A small leadingedge separation bubble was observed over the upper surface at $\alpha=16.2^{\circ}$ (Fig. 7(b)). This separated flow reattaches on the surface soon at $x=0.05$. Increasing the angle of attack increases the adverse pressure gradient and this causes the flow reattachment point moves downstream towards the trailing edge and separation bubble grows in size (Figs. 7(c), (d)). This separation bubble is responsible for the onset of the dynamic stall phenomenon. Fig. 7(e) shows that at $\alpha=18.6^{\circ}$ a vortex was formed near the leading edge, so-called dynamic stall vortex. A considerable negative wall shear stress can be seen inside dynamic stall vortex as shown in wall shear stress graphs in Figs. 7(e)-(h). The formation of dynamic stall vortex is the onset of major changes in the aerodynamic coefficients. The lift suddenly starts to increase due to augmented lift generated by the vortex. As angle of attack increases further, the dynamic stall vortex begins to move away from the leading edge area and the lift keeps increasing (Figs. 7(e)-(h)). After vortex reaches the trailing edge, the vortex lifts off the airfoil and is shed into the wake with a consequence that flow suddenly separates on the upper surface and the lift decreases very rapidly.

The unsteady lift trend follows a different path during downstroke from upstroke [47] as shown in Fig. 6(a). The instantaneous streamlines and upper surface wall shear stress during downstroke are shown in Fig. 8. As the angle of attack decreases from maximum angle of attack, some weak secondary vortices start to form and break up over the upper surface (Figs. 8(a)-(f)). The reader is referred to the graphs of wall-shear stress during the downstroke and upstroke (Figs. 7 and 8) to compare the strength of secondary vortices with dynamic stall vortex. The secondary vortices make the unsteady lift during downstroke very nonlinear as shown in Fig. $6(\mathrm{a})$. At $\alpha=15.3^{\circ}$ the separation point is at $x=0.1$ and then this point moves slowly away toward the trailing edge with further fall in the angle of attack. Even at $\alpha=9.5^{\circ}$ there are still separated flow at $x=0.4$. The identified separation points during the upstroke and downstroke are shown in Fig. 6(b). 


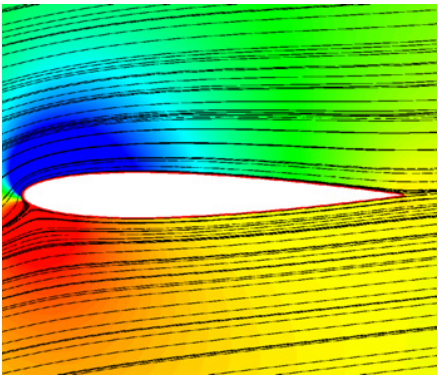

(a) $\Delta t^{*}=0$

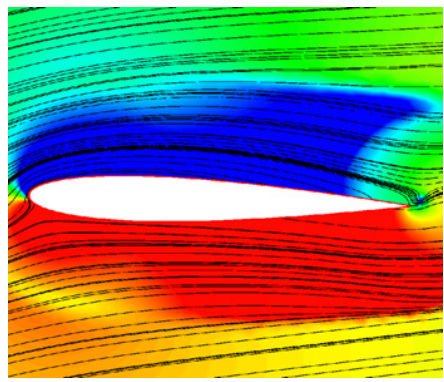

(e) $\Delta t^{*}=0.098$

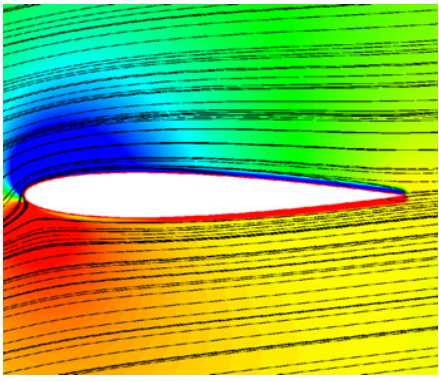

(b) $\Delta t^{*}=0.023$

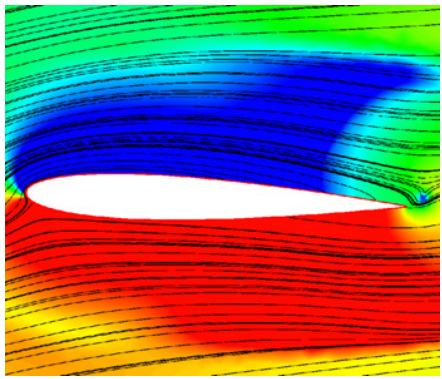

(f) $\Delta t^{*}=0.12$

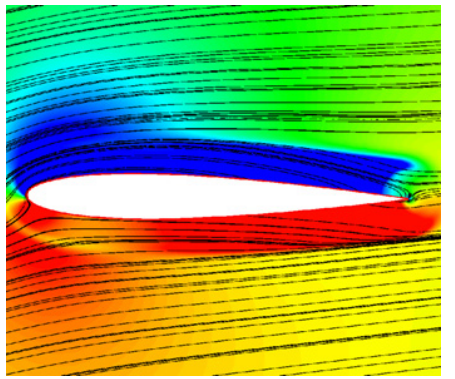

(c) $\Delta t^{*}=0.048$

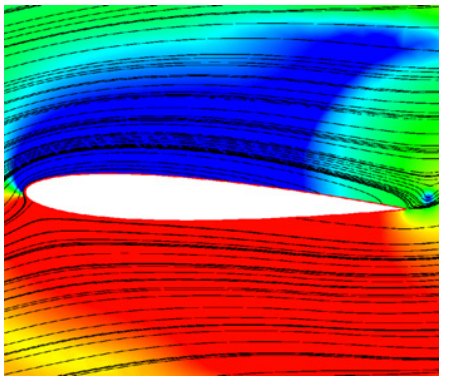

(g) $\Delta t^{*}=0.15$

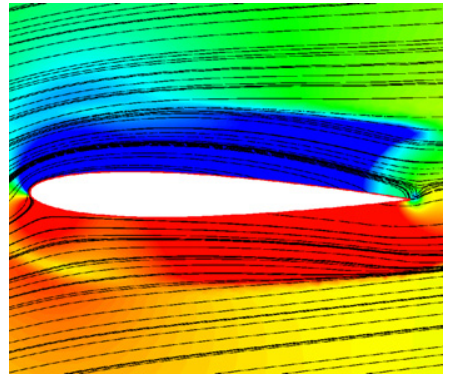

(d) $\Delta t^{*}=0.073$

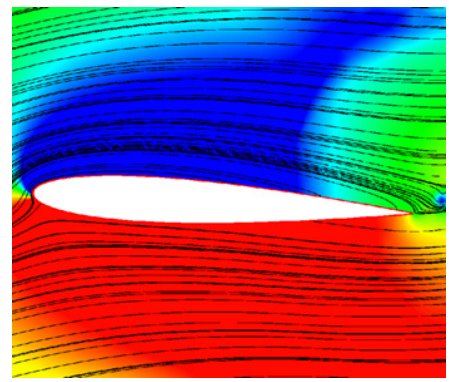

(h) $\Delta t^{*}=0.17$

Fig. 9. Initial solutions of a pitching-up airfoil: $M=0.3$ and $k=1.0$.

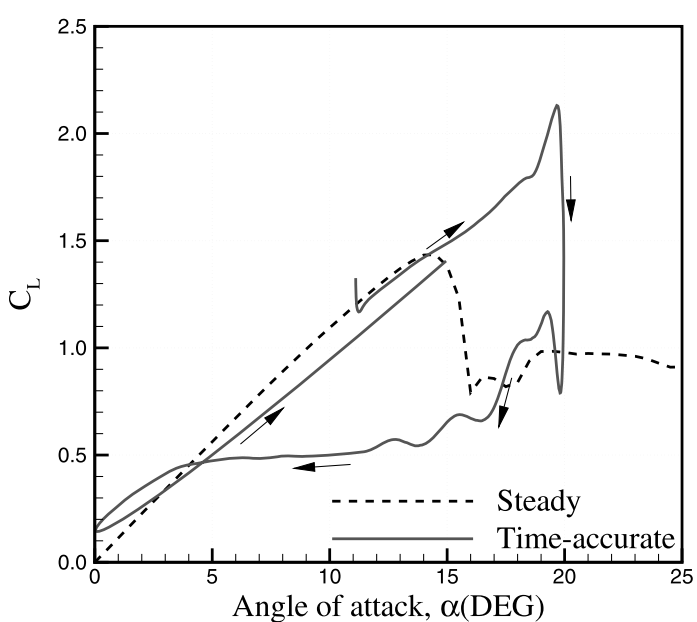

(a) Lift coefficient $-k=0.1$

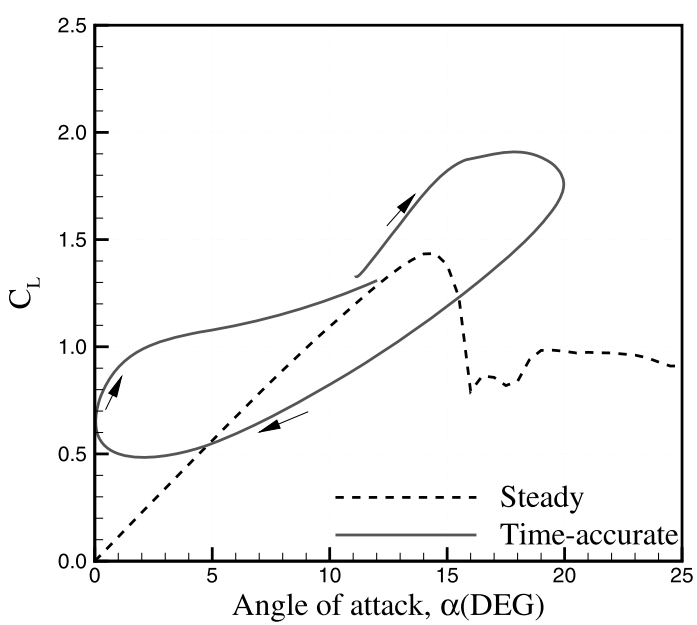

(c) Lift coefficient $-k=1.0$

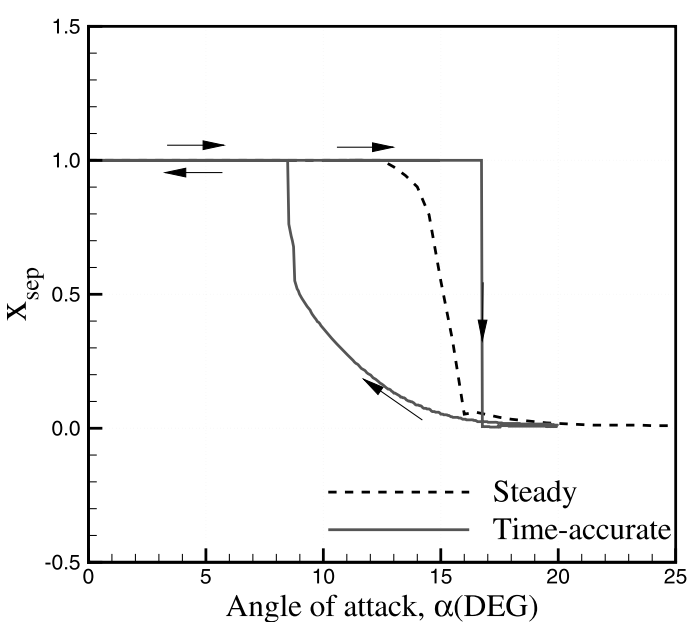

(b) Separation points $-k=0.1$

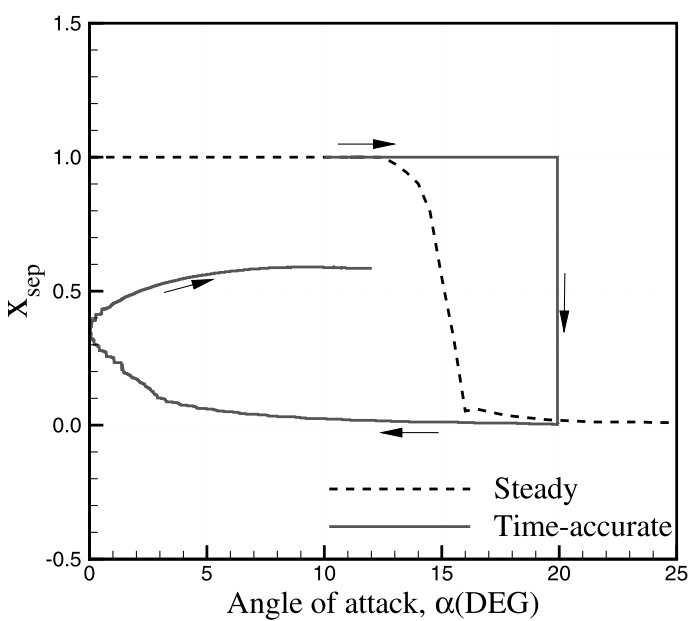

(d) Separation points $-k=1.0$

Fig. 10. Plunging airfoil: $M=0.3$. 


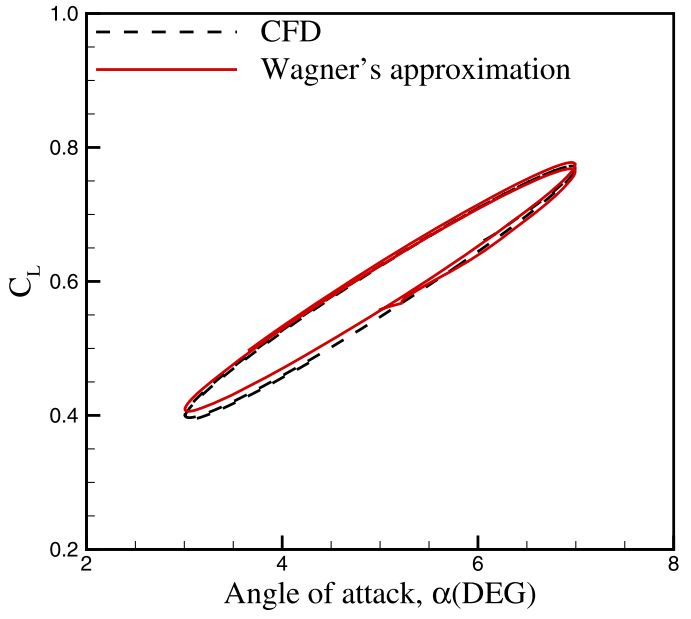

(a) $\alpha_{e}=5^{\circ}+2^{\circ} \sin (\omega t)$

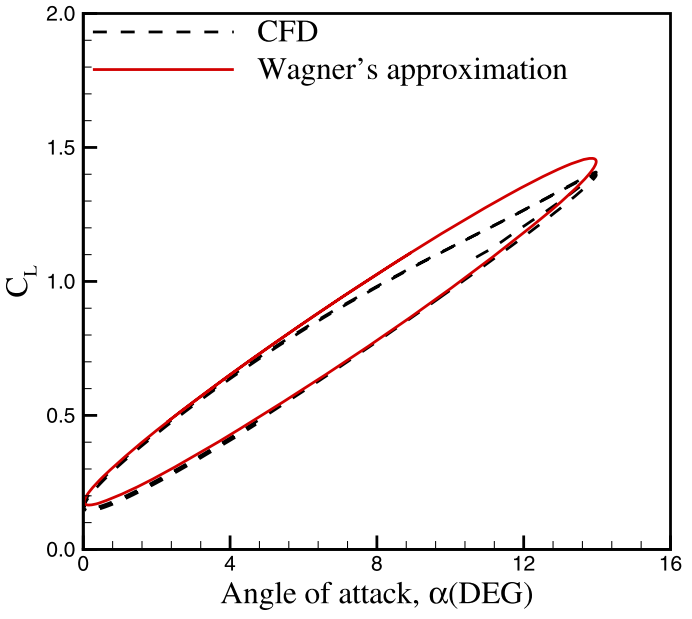

(b) $\alpha_{e}=7^{\circ}+7^{\circ} \sin (\omega t)$

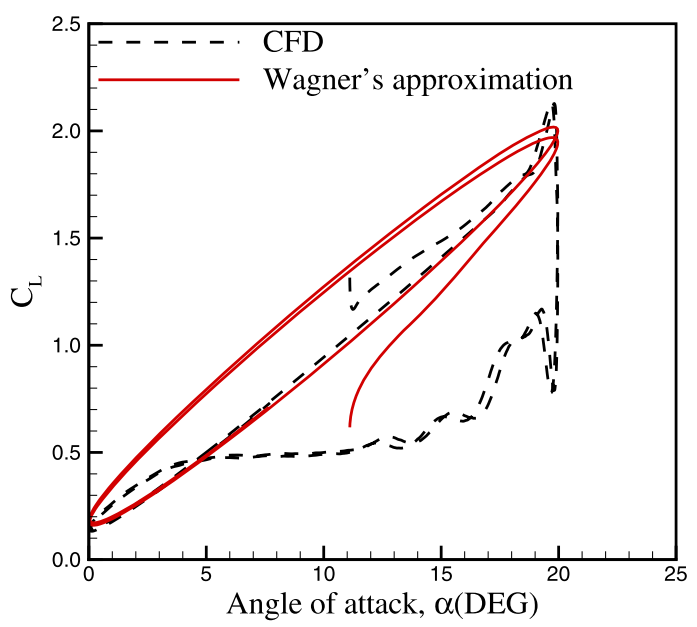

(c) $\alpha_{e}=10^{\circ}+10^{\circ} \sin (\omega t)$

Fig. 11. Modeling CFD results of plunge motions with $k=0.1$ and $M=0.3$ using Wagner's approximation.

Fig. 6(c) shows the hysteresis loop for a pitch with $k=1.0$; it can be seen that the loop is significantly different from the pitch with $k=0.1$. This very fast pitching motion have been seen in the flapping wings of an insect [52]. Such a rapid motion induces a special mechanism of flow separation and vortical flows that temporarily enhance the lift and decrease pitching moment [24, 5]. Similar aerodynamic features also occur when a vehicle impulsively starts from rest, resulting in a sudden jump in aerodynamic loads [18]. Fig. 6(d) shows the identified separation points for this fast motion; the flow separation has been delayed and occurs at maximum incidence in the cycle. The lift is significantly enhanced during upstroke; this sudden increase in the lift can be explained based on the energy of acoustic wave systems created by the initial perturbation [39]. The instantaneous streamlines and surface pressures at initial times are also shown in Fig. 9. The figure shows that no dynamic stall is formed during the upstroke, but acoustic wave are formed near the surface that temporally induce a large negative pressure over the upper surface. As the motion time increases the waves begin to move away from the surface. The wave speed depends on the flow Mach number such that it is moving slower for lower Mach numbers. As the airfoil starts to pitch down, the flow suddenly separates from the leading edge and the lift falls.

Also Fig. 10 shows the solutions of plunging motions with $k=0.1$, and $k=1$. Note that these motions have no rotation, but angle of attack changes due to grid vertical displacement. The variation of this angle of attack is identical to the pitching motion. Figs. 10(b)-(d) depict the identified separation point variation with angle of attack in a plunging motion with $k=0.1$ and $k=1.0$, respectively. The figures show that the trend of separation point are identical with those found from pitching motions. The pitch rate, $q$ is zero in the plunging motions, and hence the results confirm that separation point depends on $\dot{\alpha}$ but not $q$.

ROMs are created for each of the reduced methods and the validity of each model is tested for several plunging motions. Fig. 11 compares the full-CFD solutions with the predictions based on Wagner's approximation. The motions have a reduced frequency of $k=0.1$ but with different amplitudes. The comparisons show that the predictions match only for small angles of attack. For oscillations at large angles of attack, the loop directions are different as well. CFD shows a clockwise loop but an anticlockwise loop was predicted from Wagner's approximation. The indicial responses of the airfoil with a unit step change of angle of attack is shown in Fig. 12, where the indicial functions per radian are plotted against non-dimensional time. The lift has a peak at $s=0$ followed by a rapid falling trend. The lift again builds up and asymptotically reaches the steady-state value. The initial peak can be explained based on the energy of acoustic wave systems created by the initial perturbation [39,34]. Fig. 12(a) shows that the lift magnitudes at the initial and final time of the response are favorably compa- 


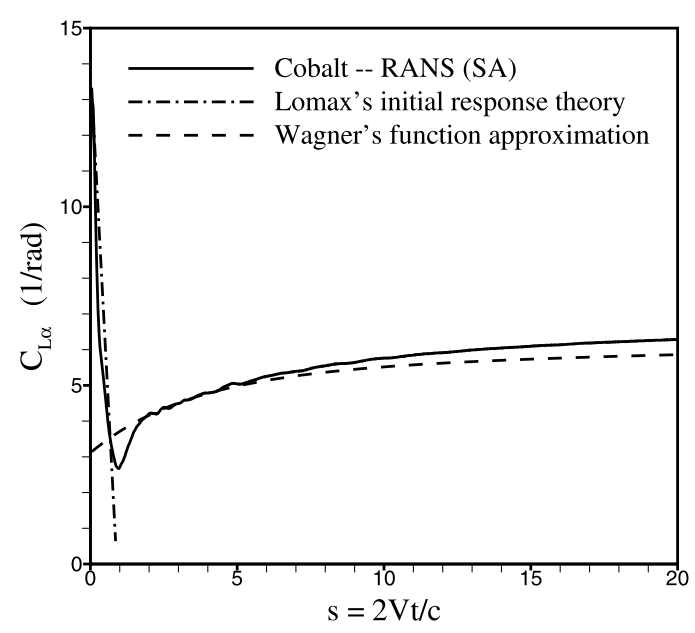

(a) linear response, $M=0.3$

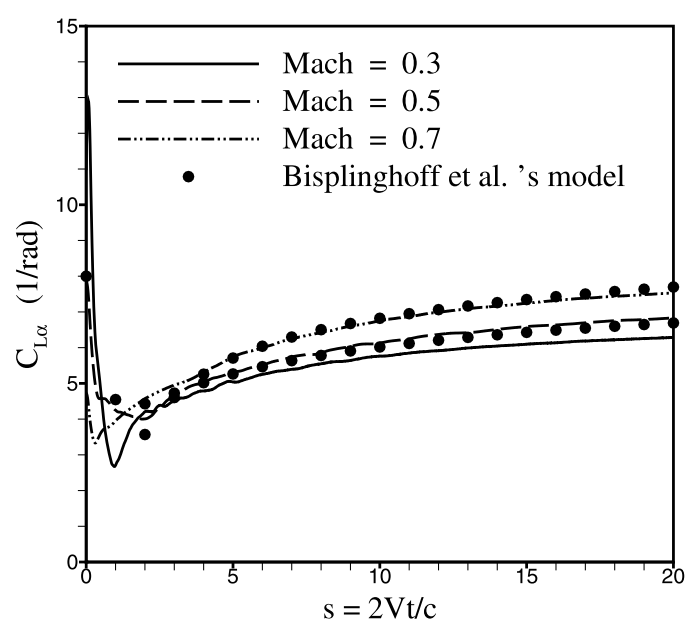

(b) linear responses at different $M$

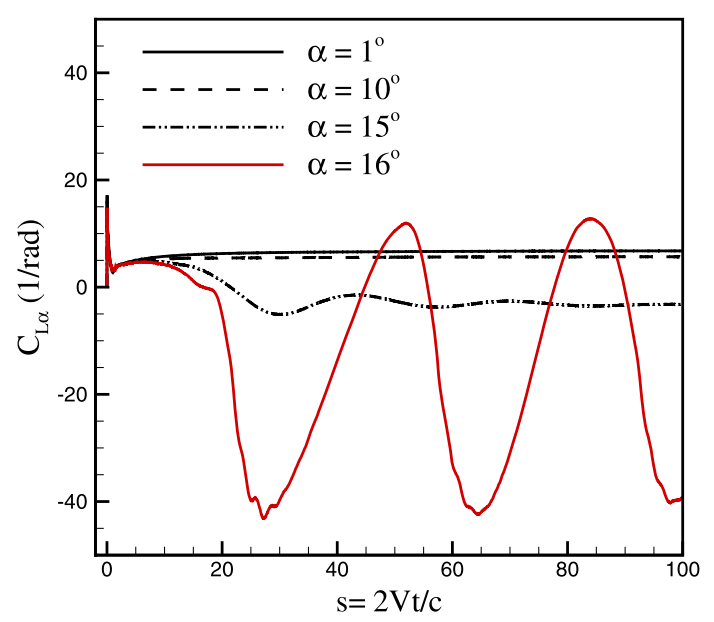

(c) nonlinear responses, $M=0.3$

Fig. 12. Indicial functions calculated directly from CFD. The Bisplinghoff et al.'s model [4] correspond to $M=0.5$ and $M=0.7$.

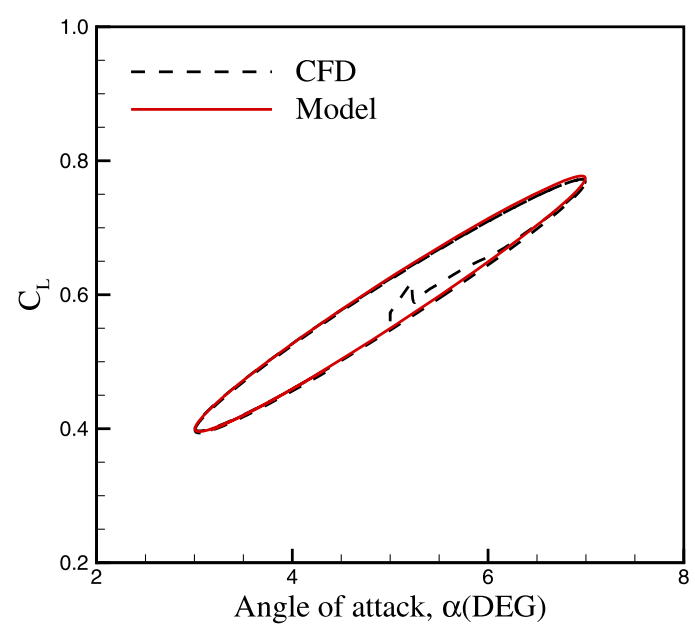

(a) $\alpha_{e}=5^{\circ}+2^{\circ} \sin (\omega t)$

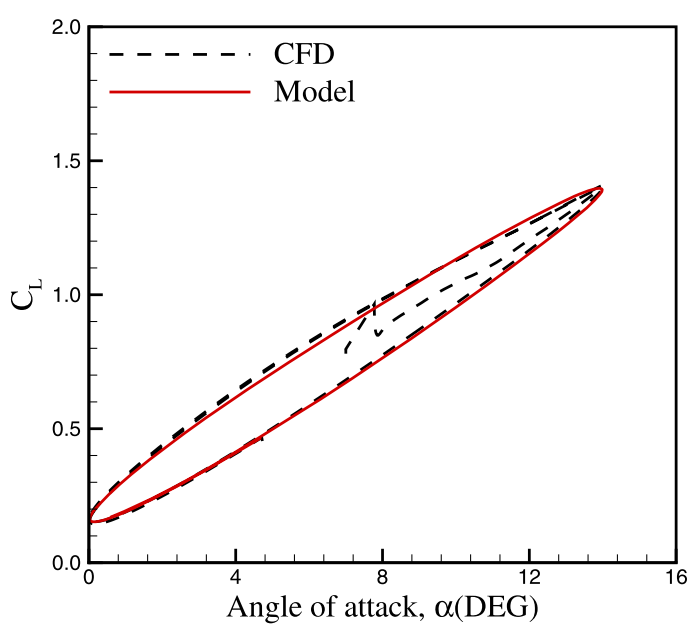

(b) $\alpha_{e}=7^{\circ}+7^{\circ} \sin (\omega t)$

Fig. 13. Modeling CFD results of plunge motions with $k=0.1$ and $M=0.3$ using indicial theory. 
rable with the analytical results of Lomax [39] and Wagner [64]. Also, the effects of Mach number for response functions are shown in Fig. 12(b). The most obvious difference is that the initial peak becomes smaller for compressible flow. An explanation is given by Leishman [34]: this is due to the propagation of pressure disturbances at the speed of sound, compared to the incompressible

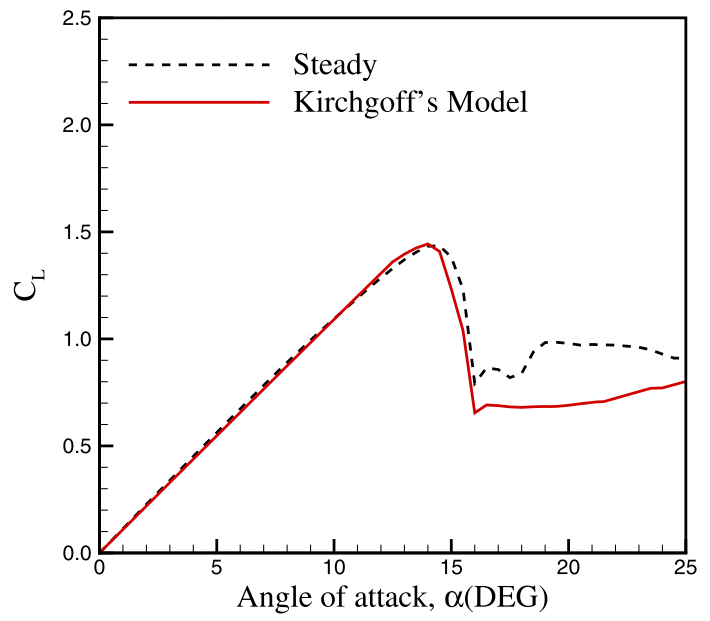

Fig. 14. Kirchgoff's model for predicting steady lift coefficient.

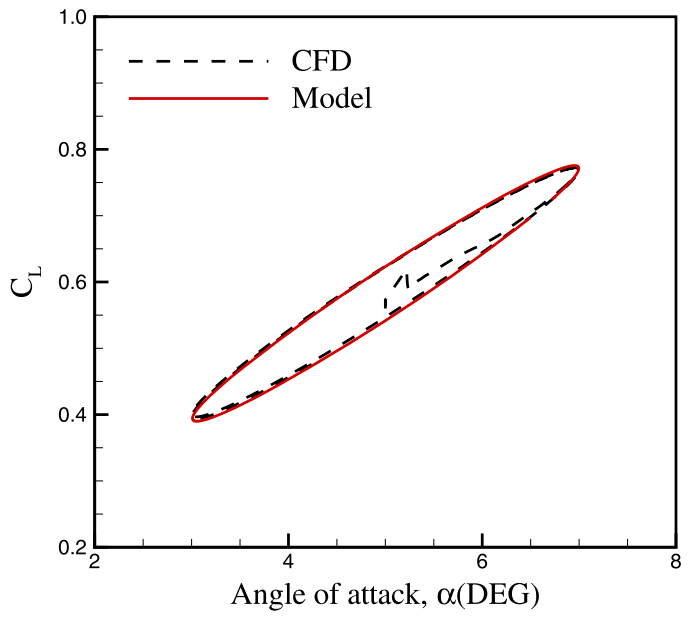

(a) $\alpha_{e}=5^{\circ}+2^{\circ} \sin (\omega t)$ case, where the disturbances propagate at infinite speed. For high incidence motions, the effects of angle of attack on indicial functions need to be determined. There are two problems: (1) a larger number of simulations is required to model a highly nonlinear motion, therefore, the reduced order model becomes very expensive to generate (2) second problem is related to the stability of response type CFD calculations at large angles of attack. Fig. 12(c) shows the effects of angle of attack into responses of NACA 0012 airfoil. It can be seen that initial responses are independent of angle of attack, but the final solutions vary depending on the angle of attack. As angle of attack reaches the stall angle, small oscillations were found in the response solutions. For angles of attack beyond the stall angle, the oscillations become large and persist for a long time. This behavior makes the nonlinear indicial functions infeasible to model motions at large incidence angles. For the motions with an amplitude below stall angle, Fig. 13 compares the predictions using a ROM based on indicial functions with CFD solutions. The figure shows that the ROM provides very good agreement for the plunging motions.

A ROM is created using Eq. (29) and was initially used for the prediction of steady solutions as shown in Fig. 14. The comparison shows a good agreement between model and CFD solutions for small to moderate angles of attack. Next, the unsteady separation point locations in the model are found using the method described above. Fig. 15 shows that predictions only match for small angles of attack but do not match well for oscillation at high angles of

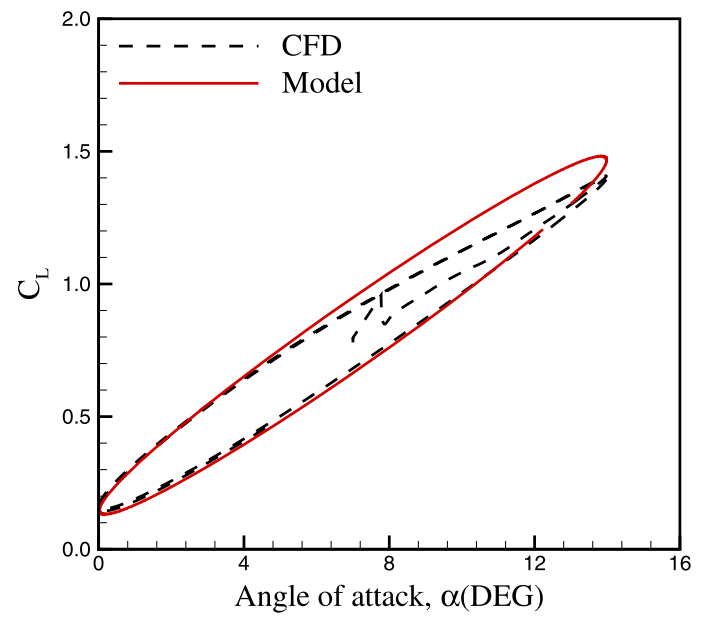

(b) $\alpha_{e}=7^{\circ}+7^{\circ} \sin (\omega t)$

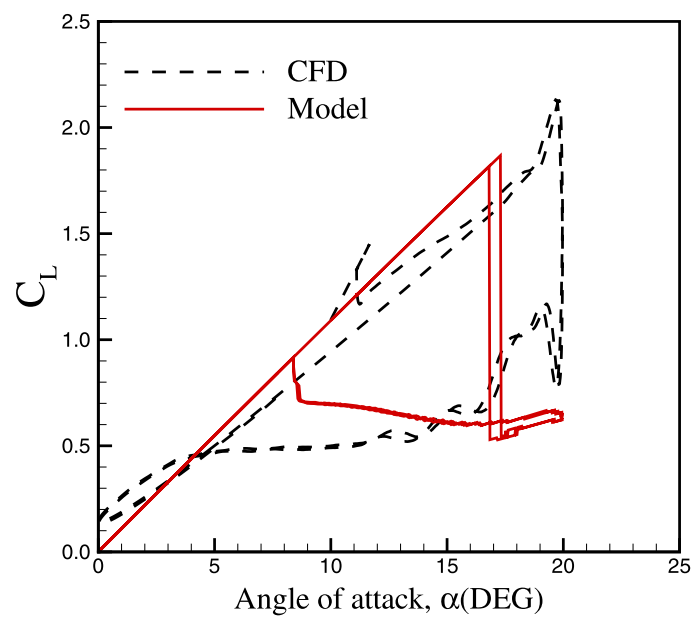

(c) $\alpha_{e}=10^{\circ}+10^{\circ} \sin (\omega t)$

Fig. 15. Modeling CFD results of a plunge motion with $k=0.1$, and $M=0.3$ using a state-space method. 


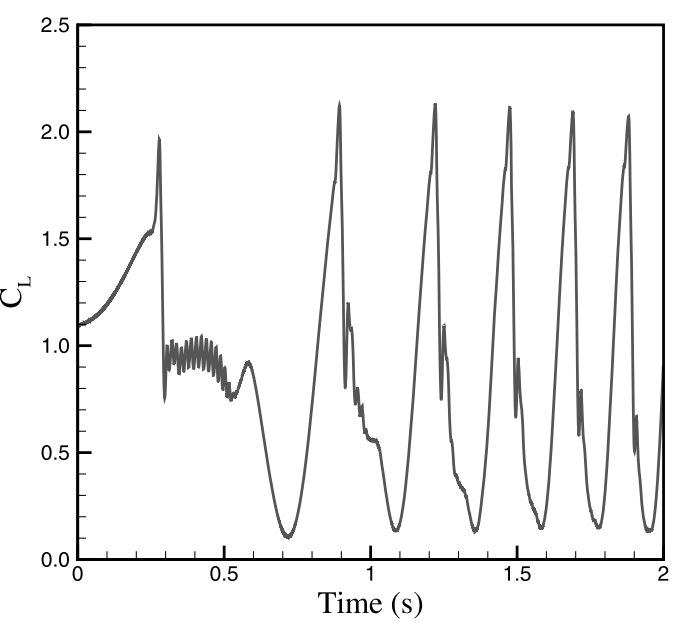

(a) chirp training maneuver

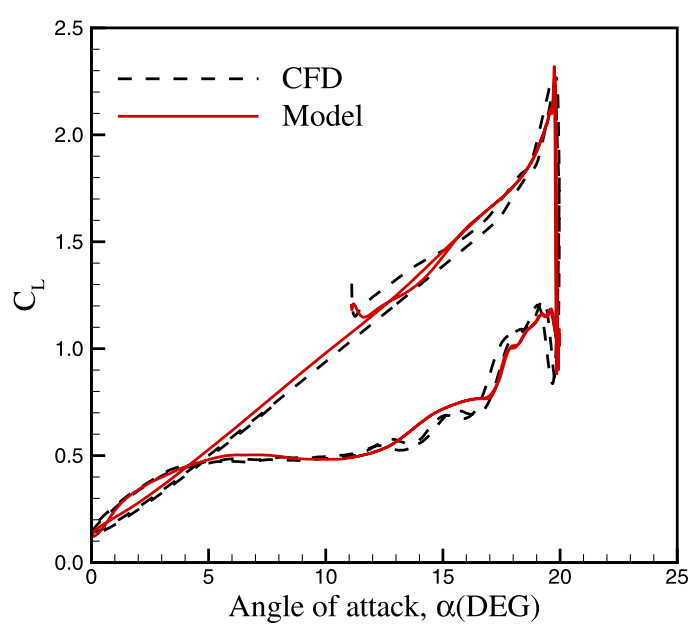

(b) prediction of plunge oscillation with $k=0.1$

Fig. 16. Modeling CFD results of a plunge motion with $k=0.1$, and $M=0.3$. The model corresponds to Radial Basis Functions with a chirp training motion.

attack. Note that Eq. (28) is valid for quasi-steady flow conditions and therefore cannot predict the effects of vortices into lift coefficient. A new mapping between lift coefficient and input variables of $\alpha$ and $x$ is suggested. This mapping can be learnt from some training maneuvers.

Finally, a chirp with linearly increasing frequency during time was defined and simulated using RANS. The maneuver is shown in Fig. 16(a); it starts from zero degrees angle of attack, has an amplitude of $10^{\circ}$ and runs for two seconds of time. The frequency linearly increases with time that causes the effects of secondary vortices into lift coefficient begin to disappear during time. The unsteady lift coefficients of chirp maneuver were re-arranged according Eq. (22) and Radial Basis Functions were used to estimate function $\Phi$. The mapping is then used to predict the results of plunging motion with $k=0.1$. The results are compared to the ROM predictions in Fig. 16(b). Figure shows that ROM prediction match CFD quite well.

\section{Conclusions}

The CFD modeling of unsteady lift coefficient of a twodimensional NACA 0012 airfoil oscillating and translating at large incidence angles was investigated in this paper. The validation results show that unsteady RANS equations are reasonable simplifications for modeling these motions. The CFD results show that a hysteresis loop was developed in the lift coefficient variation with angle of attack. Shapes of the loops change significantly with reduced frequency value. A dynamic stall vortex was identified in the pitching and plunging motions with $k=0.1$. This vortex delays the onset of flow separation over the upper surface to a higher incidence than would occur in steady conditions. The unsteady lift of very fast motions is significantly enhanced due to formation of acoustic waves. The results show clear limitations of unsteady aerodynamic theories for modeling motions at high incidences. Also, the CFD solution of indicial functions show large oscillation at angles of attack beyond the stall angle, but the predictions of a model based on Radial Basis Function matches CFD values quite well. Future work will extend this study to include more aerodynamic models and DES simulations.

\section{Acknowledgements}

Mehdi Ghoreyshi was supported by the National Research Council/US Air Force Office of Scientific Research; their financial support is gratefully acknowledged. Acknowledgements are expressed to the DoD Arctic Region Supercomputing Center (ARSC) for providing computer time. Special thanks to Bill Strang and Bob Tomaro at Cobalt Solutions to provide us a beta version of Cobalt for separation point extraction. The authors appreciate the support provided by the Modeling and Simulation Research Center at USAFA.

\section{References}

[1] M.A. Arbib, The Handbook of Brain Theory and Neural Networks, second edition, The MIT Press, 2003.

[2] R. Babuska, Fuzzy Modeling for Control, Kluwer Academic Publishers Group, Norwell, USA, 1998.

[3] W.F. Ballhaus, P.M. Goorjian, Computation of unsteady transonic flows by indicial methods, AIAA Journal 16 (2) (1978) 117-124.

[4] R.L. Bisplinghoff, H. Ashley, R.L. Halfman, Aeroelasticity, Dover, New York, 1996, pp. 350-351.

[5] S.L. Brunton, C.W. Rowley, K. Taira, T. Colonius, J. Collins, D.R. Williams, Unsteady aerodynamic forces on small-scale wings: Experiments, AIAA Paper 2008-520, January 2008.

[6] X. Chen, A. Kareem, Advances in modeling aerodynamic forces on bridge decks, Journal of Engineering Mechanics 128 (1) (2002) 1193-1205.

[7] R.M. Cummings, A. Jirásek, M. Ghoreyshi, M. Post, Numerical prediction of stability derivatives with various reduced order models, in: AVT-189 Specialists Meeting on Assessment of Stability and Control Prediction Methods for NATO Air and Sea Vehicles, Portsmouth, England, October 2011.

[8] A. Da Ronch, D. Vallespin, M. Ghoreyshi, K.J. Badcock, Evaluation of dynamic derivatives using computational fluid dynamics, AIAA Journal 50 (2) (2012) 470-484.

[9] A. Da Ronch, K.J. Badcock, M. Ghoreyshi, R.M. Cummings, Modeling unsteady aerodynamic loads, AIAA Paper 2011-6524, August 2011.

[10] T.S. Deisboeck, J.Y. Kresh, Complex Systems Science in Biomedicine, Springer, USA, 2006.

[11] W.E. Faller, S.J. Schreck, Neural networks: Applications and opportunities in aeronautics, Progress in Aerospace Sciences 32 (5) (1996) 433-456.

[12] J.R. Forsythe, S.H. Woodson, Unsteady computations of abrupt wing stall using detached-eddy simulations, Journal of Aircraft 42 (3) (2005) 606-616.

[13] J.R. Forsythe, K.A. Hoffmann, R.M. Cummings, K.D. Squires, Dettached-eddy simulation with compressibility corrections applied to a supersonic axisymmetric base, Journal of Fluids Engineering 124 (4) (2002) 911-923.

[14] R. Forsythe, K.D. Squires, K.E. Wurtzler, P.R. Spalart, Detached-eddy simulation of fighter aircraft at high alpha, Journal of Aircraft 41 (2) (2004) 193-200.

[15] A. Gaitonde, D.P. Jones, Reduced order state-space models from the pulse responses of a linearized CFD scheme, International Journal of Numerical Methods in Fluids 42 (6) (2003) 581-606.

[16] M. Ghoreyshi, A. Jirásek, R.M. Cummings, CFD modeling for trajectory predictions of a generic fighter configuration, AIAA Paper 2011-6523, August 2011.

[17] M. Ghoreyshi, D. Vallespin, A.D. Ronch, K.J. Badcock, J. Vos, S. Hitzel, Simulation of aircraft manoeuvres based on computational fluid dynamics, AIAA Paper 2010-8239, August 2010.

[18] M. Ghoryeshi, A. Jirásek, R.M. Cummings, Computational investigation into the use of response functions for aerodynamic loads modeling, AIAA Journal 50 (6) (2012) 1314-1327. 
[19] M. Ghoryeshi, A. Jirasek, M.L. Post, R.M. Cummings, Computational approximation of nonlinear unsteady aerodynamics using an aerodynamic model hierarchy, AIAA Paper 2011-3667, June 2011.

[20] M.G. Goman, A.N. Khrabov, State-space representation of aerodynamic characteristics of an aircraft at high angles of attack, AIAA Paper 92-4651, August 1992.

[21] M.G. Goman, A.N. Khrabrov, State-space representation of aerodynamic characteristics of an aircraft at high angles of attack, Journal of Aircraft 31 (5) (1994) 1109-1115.

[22] J.J. Gottlieb, C.P.T. Groth, Assessment of Riemann solvers for unsteady onedimensional inviscid flows of perfect gasses, Journal of Fluids and Structure 78 (2) (1998) 437-458.

[23] D.I. Greenwell, Frequency effects on dynamic stability derivatives obtained from small-amplitude oscillatory testing, Journal of Aircraft 35 (5) (1998) 776783.

[24] K.E. Gustafson, R. Leben, Computation of dragonfly aerodynamics, Computer Physics Communications 65 (1) (1991) 121-132.

[25] K.C. Hall, J.P. Thomas, E.H. Dowell, Reduced-order modelling of unsteady smalldisturbance using a frequency-domain proper orthogonal decomposition technique, AIAA Paper 1999-655, January 1999.

[26] D.H. Hodges, G.A. Pierce, Introduction to Structural Dynamics and Aeroelasticity, Cambridge University Press, 2002.

[27] R.V. Jategaonkar, Flight Vehicle System Identification, AIAA Educational Series, vol. 216, Reston, VA, 2006.

[28] T. Jeans, D. McDaniel, R.M. Cummings, W. Mason, Aerodynamic analysis of a generic fighter using delayed detached-eddy simulations, Journal of Aircraft 46 (4) (2009) 1326-1339.

[29] A. Jirásek, T.L. Jeans, M. Martenson, R.M. Cummings, K. Bergeron, Improved methodologies for maneuver design of aircraft stability and control simulations, AIAA Paper 2010-515, January 2010.

[30] S.G. Krantz, H.R. Parks, The Implicit Function Theorem: History, Theory, and Applications, Birkhäuser, Boston, 2002.

[31] H.G. Küssner, Zusammenfassender Bericht über den Instationaren Auftrieb von Flugeln, Luftfahrtforschung 13 (12) (1939) 410-424.

[32] H. Kyle, M. Lowenberg, D. Greenwell, Comparative evaluation of unsteady aerodynamic modeling approaches, AIAA Paper 2004-5272, August 2004

[33] C.L. Ladson, Effects of independent variation of Mach and Reynolds number on the low-speed aerodynamic characteristics of NACA0012 airfoil section, NASA Technical Paper 4074, January 1988.

[34] L.G. Leishman, Indicial lift approximations for two-dimensional subsonic flow as obtained from oscillatory measurements, Journal of Aircraft 30 (3) (1993) 340-351.

[35] J.G. Leishman, Subsonic unsteady aerodynamics caused by gusts using the indicial method, Journal of Aircraft 33 (5) (1996) 869-879.

[36] J.G. Leishman, K.Q. Nguyen, State-space representation of unsteady airfoil behavior, AIAA Journal 28 (5) (1989) 836-844.

[37] A.U. Levin, K.S. Narendra, Control of nonlinear dynamical systems using neural networks: Controllability and stabilization, IEEE Transactions on Neural Networks 4 (2) (1993) 192-206.

[38] R. Liebe, Flow Phenomena in Nature: A Challenge to Engineering Design, WIT Press, Great Britain, 2007.

[39] H. Lomax, Indicial aerodynamics, Tech. rep., AGARD Manual of Aeroelasticity, Part 2, Chapter 6, Oct. 1960.

[40] D.J. Lucia, P.S. Beran, W.A. Silva, Reduced-order modeling: New approaches for computational physics, Progress in Aerospace Sciences 40 (1) (2004) 51-117.

[41] C.E. Manglano-Villamarine, S.T. Shaw, Three-dimensional indicial response of finite aspect ratio yawed wings, The Aeronautical Journal 111 (1120) (2007) 359-371.

[42] K.W. McAlister, L.W. Carr, W.J. McCroskey, Dynamic stall experiments on the NACA 0012 airfoil, NASA Technical Paper 1100, January 1978.
[43] W.J. McCroskey, The phenomenon of dynamic stall, Technical Memo, NASA TM81264, March 1981.

[44] W.J. McCroskey, L.W. Carr, K.W. Mcalister, Dynamic stall experiments on oscillating airfoils, AIAA Journal 14 (1) (1975) 57-63.

[45] S.A. Morton, J.R. Forsythe, A.M. Mitchell, D. Hajek, Detached-eddy simulations and Reynolds-averaged Navier-Stokes simulations of delta wing vortical flowfields, Journal of Fluids Engineering 124 (4) (2002) 924-932.

[46] B.N. Pamadi, Performance, Stability, Dynamics, and Control of Airplanes, AIAA Education Series, Reston, Virginia, 2004.

[47] J. Peinke, P. Schaumann, S. Barth, Wind Energy: Proceedings of the Euromech Colloquium, Springer, Berlin, Heidelberg, New York, 2007.

[48] W.F. Phillips, Mechanics of Flight, John Wiley \& Sons, Hoboken, New Jersey, 2004.

[49] D.E. Raveh, Reduced-order models for nonlinear unsteady aerodynamics, AIAA Journal 39 (8) (2001) 1417-1429.

[50] P.H. Reisenthel, Development of a nonlinear indicial model using response functions generated by a neural network, AIAA Paper 97-0337, January 1997.

[51] P.H. Reisenthel, M.T. Bettencourt, Data-based aerodynamic modeling using nonlinear indicial theory, AIAA Paper 99-0763, January 1999.

[52] S.P. Sane, The aerodynamics of insect flight, The Journal of Experimental Biology 206 (2003) 4191-4208.

[53] H. Schilchting, K. Gersten, Boundary Layer Theory, Springer-Verlag, Berlin, Heidelberg, 2000.

[54] W. Silva, Identification of nonlinear aeroelastic systems based on the Volterra theory: Progress and opportunities, Nonlinear Dynamics 39 (1-2) (2005) 2562.

[55] W.A. Silva, Discrete-time linear and nonlinear aerodynamic impulse responses for efficient CFD analysis, PhD Dissertation, Faculty of the Department of Applied Science, The College of William and Mary in Virginia, VA, September 1997.

[56] W.A. Silva, R.E. Bartels, Development of reduced-order models for aeroelastic analysis and flutter prediction using the CFL3Dv6.0 code, Journal of Fluids and Structure 19 (6) (2004) 729-745.

[57] R. Singh, J. Baeder, Direct calculation of three-dimensional indicial lift response using computational fluid dynamics, Journal of Aircraft 34 (4) (1997) 465-471.

[58] W.Z. Strang, R.F. Tomaro, M.J. Grismer, The defining methods of cobalt: A parallel, implicit, unstructured Euler/Navier-Stokes flow solver, AIAA Paper 19990786, January 1999.

[59] A. Surana, G.B. Jacobs, G. Haller, Extraction of separation and attachment surfaces from three-dimensional steady shear flows, AIAA Journal 45 (6) (2007) 129-1302.

[60] T. Theodorsen, General theory of aerodynamic instability and the mechanism of flutter, Technical Report 496, NACA, 1935.

[61] M. Tobak, G.T. Chapman, Nonlinear problems in flight dynamics involving aerodynamic bifurcations, NACA TN-86706, November 1985.

[62] M. Tobak, G.T. Chapman, L.B. Schiff, Mathematical modeling of the aerodynamic characteristics in flight dynamics, NACA TN-85880, January 1984.

[63] R.F. Tomaro, W.Z. Strang, L.N. Sankar, An implicit algorithm for solving time dependent flows on unstructured grids, AIAA Paper 97-0333, January 1997.

[64] H. Wagner, Üeber die Entstehung des dynamischen Auftriebes von Tragflügeln, Zeitschrift für angewandte Mathematik und Mechanik 1 (1) (1925) 17-35.

[65] P.B. Walker, Experiments on the growth of circulation about a wing and an apparatus for measuring fluid motion, Rep. Memo. Aeronaut. Res., No. 1402 1931.

[66] K. Willcox, J. Peraire, Balanced model reduction via the proper orthogonal decomposition, AIAA Journal 40 (11) (2002) 2323-2330.

[67] J.R. Wright, J.E. Cooper, Introduction to Aircraft Aeroelasticity and Loads, John Wiley \& Sons Ltd., UK, 2007. 